\title{
Semiclassical trace formulas for pitchfork bifurcation sequences
}

\author{
J. Kaidel* and M. Brack \\ Institute for Theoretical Physics, University of Regensburg, D-93040 Regensburg, Germany
}

(Received 31 October 2003; published 15 July 2004)

\begin{abstract}
In nonintegrable Hamiltonian systems with mixed phase space and discrete symmetries, sequences of pitchfork bifurcations of periodic orbits pave the way from integrability to chaos. In extending the semiclassical trace formula for the spectral density, we develop a uniform approximation for the combined contribution of pitchfork bifurcation pairs. For a two-dimensional double-well potential and the familiar Hénon-Heiles potential, we obtain very good agreement with exact quantum-mechanical calculations. We also consider the integrable limit of the scenario which corresponds to the bifurcation of a torus from an isolated periodic orbit. For the separable version of the Hénon-Heiles system we give an analytical uniform trace formula, which also yields the correct harmonic-oscillator SU(2) limit at low energies, and obtain excellent agreement with the slightly coarse-grained quantum-mechanical density of states.
\end{abstract}

DOI: 10.1103/PhysRevE.70.016206

PACS number(s): 05.45.Mt

\section{INTRODUCTION}

The goal of the research area called "quantum chaos" is to relate the quantum-mechanical and classical properties of a classically chaotic system. For autonomous Hamiltonian systems, the eigenvalue spectrum is, to the leading orders in $\hbar$, dominated by the periodic orbits of the classical system. For chaotic systems, the periodic orbits are isolated in phase space and contribute individually to the semiclassical spectral density [1,2], while in integrable systems the leading contributions come from families of degenerate orbits forming rational tori $[3,4]$. The most general case is that of a system which is neither integrable nor ergodic but exhibits a mixed phase space consisting of regular islands separated by chaotic domains. The chaotic regions increase through the destruction of rational tori when continuous symmetries are broken, and through bifurcations of periodic orbits when the energy or another control parameter of the system is increased. Explicit semiclassical trace formulas have been given for various systems with continous symmetries [3,5-7], for symmetry breaking through the destruction of rational tori [8-12], and for isolated bifurcations [13-15]. However, more complicated bifurcation scenarios which usually occur in realistic physical systems and, in particular, bifurcation cascades [16-19] still constitute one of the most serious problems of the semiclassical theory.

Periodic orbits contribute to the semiclassical density of states individually only as long as they remain isolated in phase space, i.e., as long as their actions differ by large multiples of $\hbar$. Near bifurcations this condition is violated and the standard remedy is to determine a collective contribution of all periodic orbits participating in the bifurcation. In the neighborhood of a bifurcation, this was achieved in Refs. $[8,9]$ using the theory of normal forms based on the classification [20,21] of generic bifurcations with codimension 1 (i.e., bifurcations occurring when one control parameter is varied) [22]. In all these classes, a central orbit of period $n$ is

\footnotetext{
*Email address: joerg.kaidel@ physik.uni-regensburg.de
}

surrounded by $m \geqslant 1$ satellite orbits of period $n m$. The corresponding generic bifurcations are called isochronous $(m$ $=1)$, period-doubling $(m=2)$, period-tripling $(m=3)$, periodquadrupling $(m=4)$, etc. The "local" uniform approximations developed in Ref. [8] fail at large distances from the bifurcations where the orbits become isolated. In Refs. [13-15] "global" uniform approximations were developed, which interpolate between the collective contribution of the orbit cluster near a bifurcation and the sum of individual contributions of the isolated orbits far from it, as correctly described by the Gutzwiller trace formula [1]. These global uniform approximations can, with minimal modifications, also be applied to nongeneric bifurcations in systems with discrete symmetries [23]. Similar global uniform approximations have also been derived for nongeneric bifurcations of codimension 2 [24,25]. Even though such bifurcations occur only when two control parameters meet the bifurcation conditions simultaneously, they are more generally of relevance because they may appear as sequences of generic bifurcations when one of the two parameters is fixed and only the other is varied [26]. In other words: when two generic bifurcations lie so close that the orbits do not become isolated between them and hence the corresponding generic codimension-one global uniform approximations cannot be used, a description using codimension 2 (or higher) becomes necessary. Such a description was first given in Ref. [25] for codimension 2 along with a classification of the possible generic bifurcation sequences according to catastrophe theory.

In the present paper we study a sequence of two successive isochronous pitchfork bifurcations of an isolated periodic orbit. This scenario which occurs in systems with discrete symmetries is not included in the classification of codimension-2 bifurcations [25] so that at present there exists no semiclassical approach for it. In fact, it may constitute the beginning of a bifurcation cascade in which this sequence is repeated infinitely often. Such a cascade can form a geometric progression reminiscent of the Feigenbaum scenario [27] (although there the bifurcations are generically period doubling), and the new periodic orbits born at the bifurca- 
tions may exhibit self-similarity properties $[18,19]$. Bifurcation cascades are frequently found in physical systems with discrete symmetries and mixed classical dynamics [16,17], so that a semiclassical approach to those situations would seem to have been required long ago. Here we develop a uniform approximation of codimension 2 for the contribution of a pair of pitchfork bifurcations to the semiclassical density of states and test it numerically by comparison with exact quantum-mechanical calculations. The agreement turns out to be very good. The degenerate limit, in which the two pitchfork bifurcations coalesce, occurs generically in integrable systems: there a whole family of degenerate orbits, forming a torus, is born from the central orbit at the bifurcation. For this case we can give analytical expressions for our uniform approximation, and numerical calculations for a separable system yield an excellent semiclassical approximation to the exact quantum-mechanical density of states.

Our paper is organized as follows. In Sec. II we present our uniform approximation, whose detailed derivation is given in Appendix A. The uniform approximation for the bifurcation of a torus from an isolated orbit in the separable limit is discussed in Sec. III, with its detailed derivation given in Appendix B. In Sec. IV we apply our results to a two-dimensional double-well potential and to the familiar Hénon-Heiles system [28], as well as to its separable version, and compare them to results of exact quantum calculations. An alternative derivation of our uniform approximation for the separable limit from Einstein-Brillouin-Keller (EBK) quantization is given in Appendix C.

\section{UNIFORM APPROXIMATION IN THE NONINTEGRABLE CASE}

The density of states of an autonomous system with Hamiltonian $H$ is given by the trace of the retarded Green function $G(E)$

$$
\begin{gathered}
g(E)=\sum_{n} \delta\left(E-E_{n}\right)=-\frac{1}{\pi}-\operatorname{Im} \operatorname{Tr} G(E), \\
G(E)=\frac{1}{E+i 0^{+}-H} .
\end{gathered}
$$

As usual, we split $g(E)$ into a smooth and an oscillating part:

$$
g(E)=\tilde{g}(E)+\delta g(E) .
$$

The smooth part $\widetilde{g}(E)$, which semiclassically is determined by all periodic orbits of the classical system with zero length [29], may either be determined by the (extended) ThomasFermi (TF) model [30] or, where this is not analytically possible, by a numerical Strutinsky averaging of the quantum spectrum [30,31]. The periodic orbits of finite length make up the oscillating part $\delta g(E)$.

The semiclassical contribution to $\delta g(E)$ of any region $\Omega$ on a Poincaré surface of section (PSS) of the phase space is given by $[9,13]$

$$
\begin{aligned}
\delta g_{\Omega}(E)= & \frac{1}{2 \pi^{2} \hbar^{2}} \operatorname{Re} \int_{\Omega} d q^{\prime} d p \frac{1}{n} \frac{\partial \hat{S}}{\partial E}\left|\frac{\partial^{2} \hat{S}}{\partial p \partial q^{\prime}}\right| \\
& \times \exp \left[\frac{i}{\hbar} \hat{S}\left(q^{\prime}, p, E\right)-\frac{i}{\hbar} q^{\prime} p-\frac{i \pi}{2} \nu\right] .
\end{aligned}
$$

Here $q^{\prime}$ are the final coordinates and $p$ the initial momenta on the PSS transverse to a periodic orbit with period $T$ centered in the origin. The $n$th iterate of the Poincare map is given by its generating function $\hat{S}\left(q^{\prime}, p, E\right)$, and the usual canonical relations hold:

$$
\frac{\partial \hat{S}}{\partial q^{\prime}}=p^{\prime}, \quad \frac{\partial \hat{S}}{\partial p}=q, \quad \frac{\partial \hat{S}}{\partial E}=T .
$$

The periodic orbits are the solutions of

$$
\frac{\partial \hat{S}}{\partial q^{\prime}}=p, \quad \frac{\partial \hat{S}}{\partial p}=q^{\prime},
$$

which are the stationary points of the phase in Eq. (3). If the integrals in Eq. (3) are calculated in the stationary-phase approximation, one obtains the individual Gutzwiller contributions of the periodic orbits $\xi$ within $\Omega$ :

$$
\delta g_{\xi}(E)=\mathcal{A}_{\xi}(E) \cos \left(\frac{S_{\xi}(E)}{\hbar}-\frac{\pi}{2} \mu_{\xi}\right),
$$

where for a two-dimensional system the amplitudes have the form

$$
\mathcal{A}_{\xi}(E)=\frac{T_{\xi}(E)}{\pi \hbar n_{\xi} \sqrt{\left|\operatorname{Tr} \tilde{M}_{\xi}-2\right|}} .
$$

The quantities $S_{\xi}, T_{\xi}, n_{\xi}, \tilde{M}_{\xi}$, and $\mu_{\xi}$ are the action, period, repetition number, stability matrix, and Maslov index of the orbit $\xi$, respectively. The stationary-phase approximation yields good results only if the periodic orbits are isolated in phase space. Near a bifurcation this condition is not fulfilled so that one has to perform the integrals in Eq. (3) collectively over the whole periodic orbit cluster involved in the bifurcation. To this purpose, one inserts the normal form of the generating function $\hat{S}\left(q^{\prime}, p, E\right)$ into Eq. (3) and solves the resulting integrals exactly.

A sequence of two period-doubling bifurcations of periodic orbits is not generic because it would imply a jump in the stability of the central periodic orbit [25]. On the other hand, an isochronous bifurcation creating a new orbit with a degeneracy factor of 2 is equivalent to a generic perioddoubling bifurcation [23]. The degeneracy factor 2 has to originate from a twofold discrete symmetry of the system. Due to the behavior of $\operatorname{Tr} \tilde{M}_{\xi}$ near the bifurcation, a generic period-doubling bifurcation is often called a pitchfork bifurcation. The case of interest here is a sequence of two such nongeneric pitchfork bifurcations which can arise succesively from the same central periodic orbit in systems with discrete symmetries such as studied in Refs. [18,19]. For this scenario we propose the new normal form 


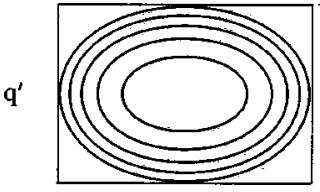

p $q^{\prime}$

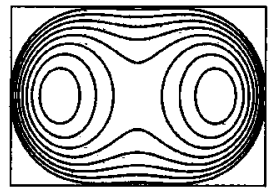

$q^{\prime}$

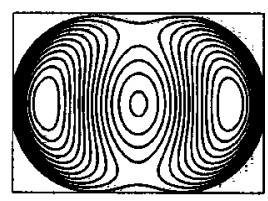

FIG. 1. Contour plots of the normal form (8) in dependence of the parameters $\epsilon_{i}$ for the case $a=-1$. From left to right: $\epsilon_{2}<\epsilon_{1}<0, \epsilon_{2}<0<\epsilon_{1}$, and $0<\epsilon_{2}<\epsilon_{1}$.

$\mathrm{p}$

$$
\begin{aligned}
\hat{S}\left(q^{\prime}, p, E\right) & =S_{0}(E)+q^{\prime} p-\frac{1}{2}\left(\epsilon_{1} p^{2}+\epsilon_{2} q^{\prime 2}\right)-\frac{a}{4}\left(p^{2}+q^{\prime 2}\right)^{2} \\
& =S_{0}(E)+q^{\prime} p-\left(\epsilon_{1} \cos ^{2} \phi+\epsilon_{2} \sin ^{2} \phi\right) I-a I^{2} .
\end{aligned}
$$

Here $q^{\prime}=\sqrt{2 I} \sin \phi$ and $p=\sqrt{2 I} \cos \phi$ define the polar coordinates $(I, \phi)$ on the PSS, with the central orbit sitting at $I$ $=0$. The parameters $\epsilon_{1}$ and $\epsilon_{2}$ measure the distance to the bifurcations and become zero at the first and second bifurcation, respectively. Inserting the angles $\phi=0$ and $\phi=\pi / 2$ into Eq. (8), one obtains the respective generic normal forms of the period-doubling bifurcations [14] corresponding to two cusp catastrophes:

$$
\hat{S}\left(q^{\prime}(0, I), p(0, I), E\right)-S_{0}(E)-q^{\prime}(0, I) p(0, I)=-\frac{\epsilon_{1}}{2} p^{2}-\frac{a}{4} p^{4}
$$

and

$$
\begin{aligned}
\hat{S}\left(q^{\prime}\left(\frac{\pi}{2}, I\right), p\left(\frac{\pi}{2}, I\right), E\right)-S_{0}(E)-q^{\prime}\left(\frac{\pi}{2}, I\right) p\left(\frac{\pi}{2}, I\right) \\
=-\frac{\epsilon_{2}}{2} q^{\prime 2}-\frac{a}{4} q^{\prime 4} .
\end{aligned}
$$

The stationary points of Eqs. (9) and (10) are the stationary points of Eq. (8) as well. The dependence of the topology of Eq. (8) on the parameters $\epsilon_{i}$ is sketched in Fig. 1.

The period-doubling bifurcations always have a real side where the central orbit as well as its satellite orbits are real, and a complex side where the central orbit is real but the satellite orbits are complex ghost orbits. We introduce a parameter $\sigma_{i}$ which is +1 on the real side and -1 on the complex side of the pitchfork bifurcation $i$ with $i=1,2$. Additionally, the sign of the difference between the actions $S_{i}$ of the new satellite orbits and the action $S_{0}$ of the central orbit is indicated by $\widetilde{\sigma}_{i} \equiv \operatorname{sgn}\left(\Delta S_{i}\right)$ and $\Delta S_{i} \equiv S_{i}-S_{0}$ with $i=1,2$.

The uniform approximation describing the contribution of the orbit cluster involved in the bifurcation sequence is derived in detail in Appendix A. It reads

$$
\begin{aligned}
\delta g(E)= & \frac{1}{4 \pi^{2} \hbar^{2}} \operatorname{Re}\left\{e^{i\left[(1 / \hbar) S_{0}-(\pi / 2) \nu\right]}\right. \\
& \left.\times \int_{0}^{2 \pi} d \phi\left[\alpha_{0} F_{0}(\phi)+\alpha_{1} F_{1}(\phi)+\alpha_{2} F_{2}(\phi)\right]\right\},
\end{aligned}
$$

where the functions $F_{i}(\phi)$ with $i=0,1,2$ are given by

$$
\begin{gathered}
F_{0}(\phi)=e^{(i / \hbar)\left(\widetilde{\sigma}_{i} / 4\right) \tilde{\epsilon}^{2}(\phi)} \sqrt{\frac{\pi \hbar}{2}}\left\{\frac{1}{\sqrt{2}} e^{-i(\pi / 4) \widetilde{\sigma}_{i}}\right. \\
\left.+\sigma\left[C\left(\sqrt{\frac{\tilde{\epsilon}^{2}(\phi)}{2 \pi \hbar}}\right)-i \widetilde{\sigma}_{i} S\left(\sqrt{\frac{\tilde{\epsilon}^{2}(\phi)}{2 \pi \hbar}}\right)\right]\right\}, \\
F_{1}(\phi)=-\frac{1}{2 \widetilde{\sigma}_{i}}\left[i \hbar+\widetilde{\epsilon}(\phi) F_{0}(\phi)\right], \\
F_{2}(\phi)=-\frac{i \hbar}{2 \widetilde{\sigma}_{i}}\left[1-\frac{\widetilde{\epsilon}(\phi)}{2 \widetilde{\sigma}_{i}}-\frac{\widetilde{\epsilon}^{2}(\phi)}{2 i \hbar \widetilde{\sigma}_{i}} F_{0}(\phi)\right],
\end{gathered}
$$

and we have used

$$
\epsilon_{i}=-2 \sigma_{i} \widetilde{\sigma}_{i} \sqrt{\left|\Delta S_{i}\right|},
$$

$$
\tilde{\epsilon}(\phi)=\epsilon_{1} \cos ^{2} \phi+\epsilon_{2} \sin ^{2} \phi,
$$

$$
\sigma=-\widetilde{\sigma}_{i} \operatorname{sgn}[\widetilde{\epsilon}(\phi)] .
$$

For the evaluation of Eqs. (12)-(14), any of the $\widetilde{\sigma}_{i}= \pm 1$ can be used, as described in Appendix A. The coefficents $\alpha_{0}, \alpha_{1}$, and $\alpha_{2}$ are given as solutions of the linear system of equations

$$
\begin{gathered}
\mathcal{A}_{0}=\frac{\alpha_{0}}{\pi \hbar \sqrt{\left|\epsilon_{1} \epsilon_{2}\right|}}, \quad \mathcal{A}_{1}=\frac{\alpha_{0}-\frac{\alpha_{1}}{2} \epsilon_{1}+\frac{\alpha_{2}}{4} \epsilon_{1}^{2}}{\pi \hbar \sqrt{\left|-2 \epsilon_{1} \epsilon_{2}+2 \epsilon_{1}^{2}\right|}}, \\
\mathcal{A}_{2}=\frac{\alpha_{0}-\frac{\alpha_{1}}{2} \epsilon_{2}+\frac{\alpha_{2}}{4} \epsilon_{2}^{2}}{\pi \hbar \sqrt{\left|-2 \epsilon_{1} \epsilon_{2}+2 \epsilon_{2}^{2}\right|}},
\end{gathered}
$$

where the amplitudes $\mathcal{A}_{i}$ with $i=0,1,2$ are given in Eq. (7). $C(x)$ and $S(x)$ are the standard Fresnel functions [32]. The index $\nu$ is related to the Maslov index $\mu_{0}$ of the central orbit by

$$
\nu=\mu_{0}-\left(\sigma_{1}+\sigma_{2}\right) / 2 .
$$

All coefficients in Eq. (11) are expressed by the quantities which appear also in the Gutzwiller contributions (6), which means that the uniform approximation is invariant under canonical transformations.

\section{UNIFORM APPROXIMATION FOR THE SEPARABLE LIMIT}

In the degenerate case $\epsilon \equiv \epsilon_{1}=\epsilon_{2}$ the normal form (8) becomes 

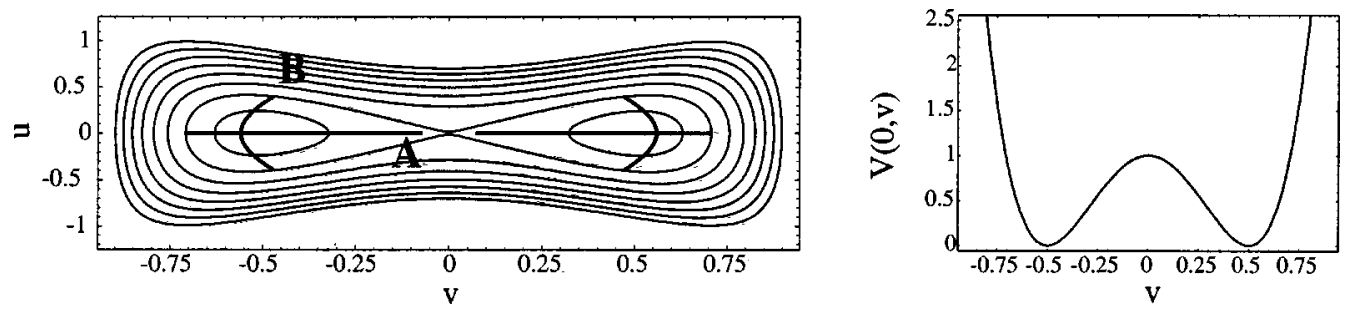

FIG. 2. Scaled double-well potential. Left: contour plot with the four shortest periodic orbits $A$ and $B$ evaluated at $e=0.96$. Right: cut of the potential along $u=0$.

$$
\begin{aligned}
\hat{S}\left(q^{\prime}, p, E\right) & =S_{0}(E)+q^{\prime} p-\epsilon\left(\frac{p^{2}+q^{\prime 2}}{2}\right)-a\left(\frac{p^{2}+q^{\prime 2}}{2}\right)^{2} \\
& =S_{0}(E)+q^{\prime} p-\epsilon I-a I^{2} .
\end{aligned}
$$

Here $\hat{S}\left(q^{\prime}, p, E\right)-q^{\prime} p$ is independent of the angle $\phi$, which means that it refers to an integrable system in which the Hamiltonian depends only on the action variables but not on the angles. The normal form (17) and the corresponding bifurcation scenario has been studied in earlier works $[8,9,15,33]$. What we intend here is to solve the necessary integrals analytically and express all the coefficients by the actions and the Gutzwiller or Berry-Tabor amplitudes of the periodic orbits, in order to give a final formula which is easy for implementation in actual examples.

The stationary point of the function $\hat{S}-q^{\prime} p$ now corresponds to a family of periodic orbits, i.e., a rational torus which is created from the central orbit at the bifurcation [9]. It consists of real periodic orbits on one side, whereas its periodic orbits have complex coordinates on the opposite side of the bifurcation. To distinguish between the two sides we introduce a parameter $\sigma$ which takes the value +1 on the side where the torus is real and -1 on the side where it is complex.

The uniform approximation for this degenerate limit $\epsilon_{1}$ $=\epsilon_{2}$ of Eq. (11) is derived in Appendix B and can be given, to the leading orders $\hbar$, in analytical form as

$$
\begin{aligned}
\delta g(E)= & \frac{\mathcal{A}_{T}}{\sqrt{2}} \operatorname{Re}\left(e ^ { i [ ( 1 / \hbar ) S _ { T ^ { - } } ( \pi / 2 ) \nu ] } \left\{\frac{1}{\sqrt{2}} e^{-i \widetilde{\sigma}(\pi / 4)}\right.\right. \\
& \left.\left.+\sigma\left[C\left(\sqrt{\frac{2|\Delta S|}{\pi \hbar}}\right)-i \widetilde{\sigma} S\left(\sqrt{\frac{2|\Delta S|}{\pi \hbar}}\right)\right]\right\}\right) \\
& +\sigma \widetilde{\sigma}\left(\mathcal{A}_{T} \sqrt{\frac{\hbar}{4 \pi|\Delta S|}}-\mathcal{A}_{0}\right) \cos \left(\frac{S_{0}}{\hbar}-\frac{\pi}{2}(\nu+1)\right),
\end{aligned}
$$

where $S_{0}$ and $S_{T}$ are the actions of the central orbit and the torus, respectively, and their difference is denoted by

$$
\Delta S \equiv S_{T}-S_{0}
$$

with $\widetilde{\sigma} \equiv \operatorname{sgn}(\Delta S)=\operatorname{sgn}(a)$. The amplitude $\mathcal{A}_{0}$ corresponds to the Gutzwiller amplitude (7) of the central periodic orbit, whereas for the torus one has to use the Berry-Tabor amplitude $\mathcal{A}_{T}[4,33]$. The Morse index $\nu$ appearing in Eq. (18) is related to the Maslov index $\mu_{0}$ of the central periodic orbit by

$$
\nu=\mu_{0}+\sigma \widetilde{\sigma}
$$

\section{NUMERICAL RESULTS}

In order to test the above uniform approximations we apply them to two model systems: (i) a double-well potential
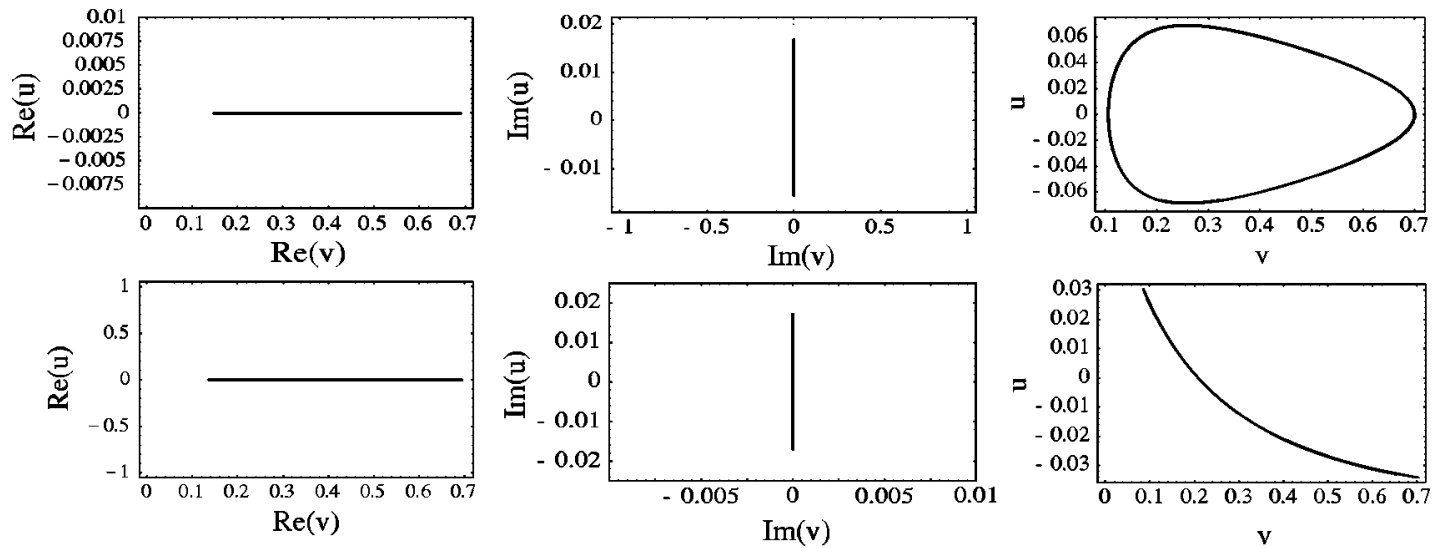

FIG. 3. Orbits participating in a pitchfork bifurcation sequence in the double-well potential (21). Upper row: real part (left) and imaginary part (middle) of ghost orbit $R$ at $e=0.908$ 64, and real orbit $R$ at $e=0.95$ (right). Lower row: real part (left) and imaginary part (middle) of ghost orbit $L$ at $e=0.94$, and real orbit $L$ at $e=0.95$ (right). 

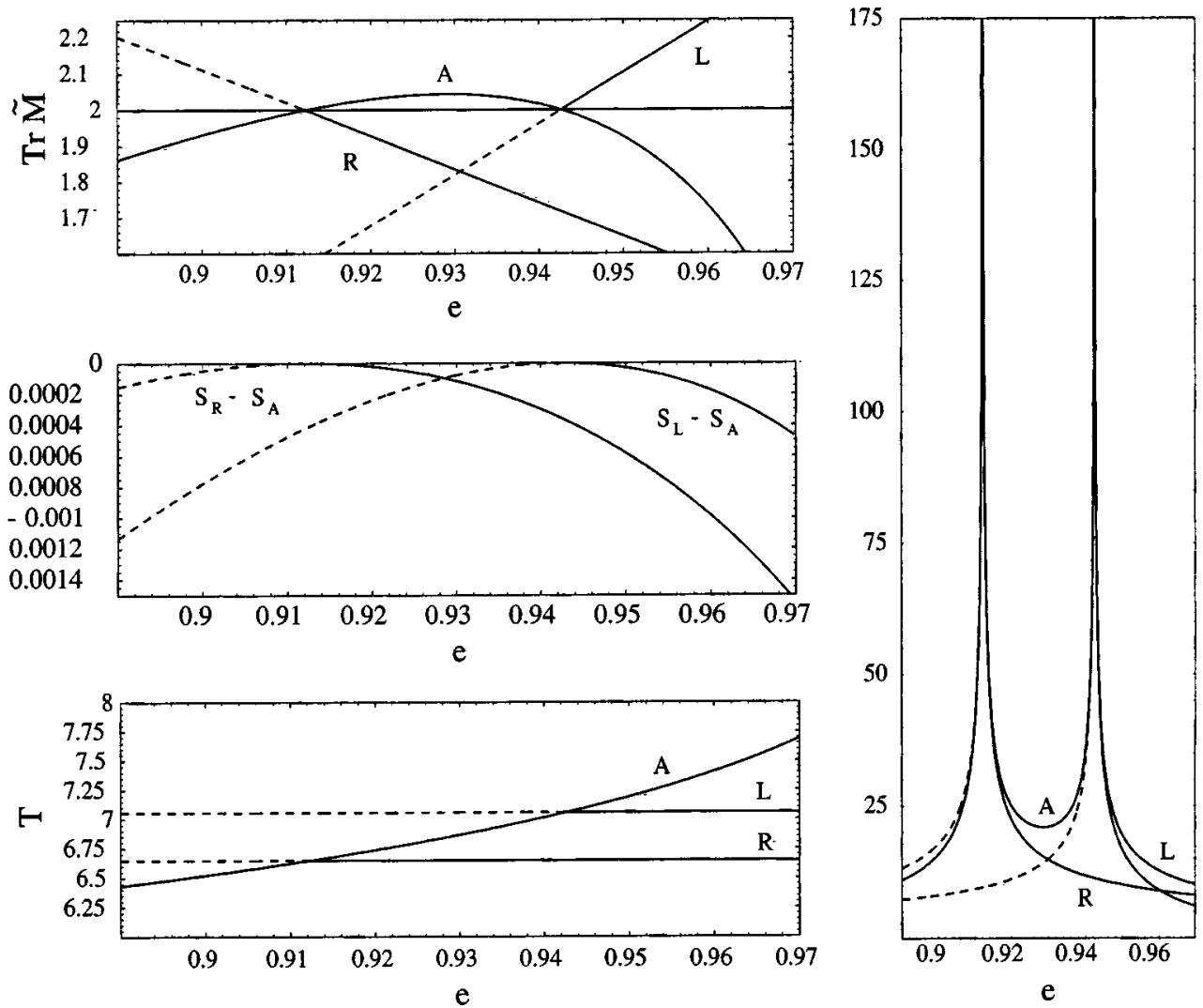

FIG. 4. Properties of the periodic orbits $A, R$, and $L$ near their bifurcations in the double-well potential (21), plotted versus the scaled energy $e$. Top left, stability traces; middle left, action differences; bottom left, periods; and right, Gutzwiller amplitudes (cf. text). The dashed portions of all curves correspond to the complex pre-bifurcation ghost orbits.

which classically possesses two bifurcation cascades, one for approaching the saddle from below and one for approaching it from above, and (ii) the familiar Hénon-Heiles system as well as its separable version. We compare with results from exact quantum calculations and discuss the validity of the uniform approximations.

\section{A. Two-dimensional double-well potential}

We study the following Hamiltonian with a double-well potential (in dimensionless units with $m=\hbar=1$ ):

$$
H=\frac{1}{2}\left(p_{x}^{2}+p_{y}^{2}\right)+\frac{1}{2}\left(x^{2}-y^{2}\right)+\lambda\left(y^{4}-\frac{1}{2} x^{2} y^{2}\right)+\frac{1}{16 \lambda} \text {. }
$$

The potential in Eq. (21) has two minima at $x=0$ and $y$ $= \pm 1 / 2 \sqrt{\lambda}$ with energy $E=0$, separated by a saddle at $x=y$ $=0$ with energy $E^{*}=1 / 16 \lambda$. Using dimensionless scaled variables $u=\sqrt{\lambda} x$ and $v=\sqrt{\lambda} y$, the classical dynamics of the system only depends on one scaled energy variable $e=E / E^{*}$ $=16 \lambda E$, with the central saddle at the height $e=1$ (see Fig. 2 ). At a scaled energy $e=9$, the system possesses four other
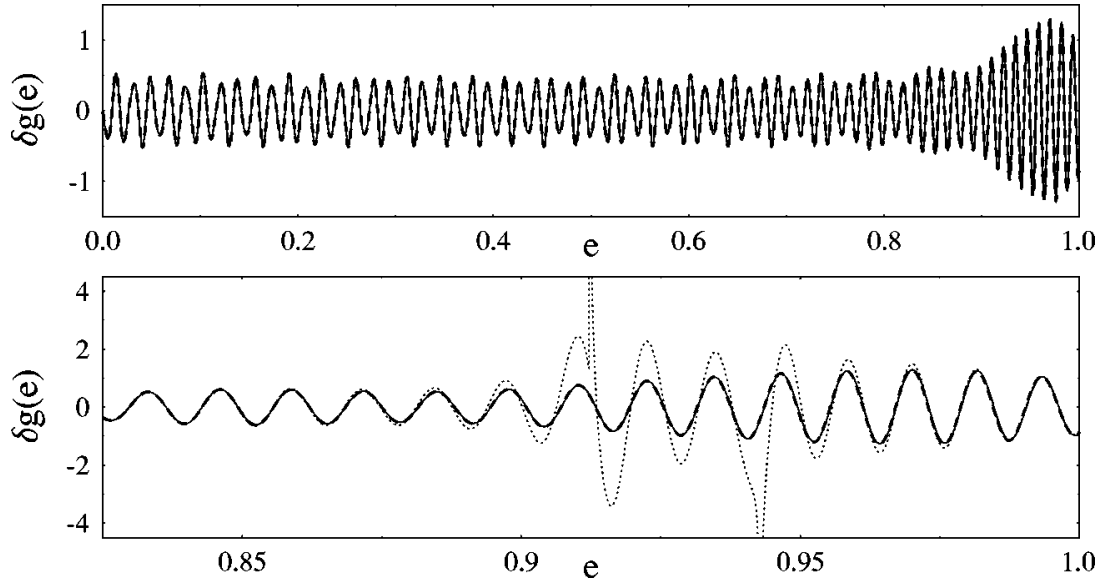

FIG. 5. Oscillating part of density of states in the double-well potential (21). Solid line: exact quantum result obtained with $\lambda$ $=0.0008$. Dashed line: uniform approximation including isolated contribution of orbit $B$. Dotted line: sum of Gutzwiller contributions of isolated orbits, diverging at the two lowest bifurcations of the $A$ orbit. (The other bifurcations, lying at $e>0.9998$, cannot be seen at this resolution.) Coarse graining by Gaussian convolution with energy width $\gamma=0.5$. 
saddles at $v= \pm 1$ and $u= \pm \sqrt{3}$, over which a particle can escape. At all energies $e>0$, there exist orbits $A$ and $B$ that librate along and across the $v$ axis, respectively. Orbit $B$ is stable up to $e=4.778$ and orbit $A$ undergoes two bifurcation cascades, one approaching the saddle at $e=1$ from below, and one approaching $e=1$ from above. We consider here only energies $e \leqslant 1$, for which all periodic orbits appear twice corresponding to the two potential wells. In this region, the influence of the continuum above $e=9$ can be safely neglected and the quantum spectrum is real and discrete to a very good approximation. We have obtained it numerically by diagonalisation of Eq. (21) in a finite harmonic-oscillator basis.

The period and action of the $A$ orbit are given analytically in terms of its two turning points, for $v>0$ given by

$$
v_{1}=\frac{1}{2} \sqrt{1-\sqrt{e}}, \quad v_{2}=\frac{1}{2} \sqrt{1+\sqrt{e}} \quad(e \leqslant 1) .
$$

The (dimensionless) period becomes

$$
T_{A}(E)=\frac{\sqrt{2}}{v_{2}} K(q),
$$

and the action is

$$
S_{A}(E)=\frac{2 \sqrt{2}}{3 \lambda} v_{2}\left[\frac{1}{2} E(q)-2 v_{1}^{2} K(q)\right],
$$

where $E$ and $K$ are the complete elliptic integrals [32] with modulus $q$ :

$$
q=\frac{1}{v_{2}} \sqrt{v_{2}^{2}-v_{1}^{2}}
$$

The dimensionless average (TF) level density of this system (including a factor 2 which accounts for the two wells) is given by the integral

$$
g_{T F}(E)=\frac{2 \sqrt{2}}{\pi \sqrt{\lambda}} \int_{v_{1}}^{v_{2}} \frac{\sqrt{\left(v_{2}^{2}-v^{2}\right)\left(v^{2}-v_{1}^{2}\right)}}{\sqrt{1-v^{2}}} d v
$$

which we could not express in a simple closed form and therefore integrated numerically.

At the energy $e=0.91232$ orbit $A$ becomes unstable, creating a stable rotational orbit $R$ with Maslov index 5. At $e$ $=0.94272$ orbit $A$ becomes stable again, creating an unstable librational orbit $L$ with Maslov index 6. In Fig. 3 the periodic orbits $R$ and $L$ are shown together with their complex "ghost" predecessors which correspond to librations in the real and imaginary parts, respectively. The bifurcation scenario is seen in the upper left panel of Fig. 4 in terms of the stability traces.

In Fig. 4 we also show the action differences and periods of the three orbits, as well as their Gutzwiller amplitudes, plotted versus the scaled energy $e$. As shown analytically in Ref. [14], the asymptotic divergences of the amplitudes of the central orbit (here $A$ ) and the satellite orbits (here $R$ and $L$ ) must differ by a factor $\sqrt{2}$; this factor has been included in the right panel of Fig. 4 in order to confirm this fact numerically.
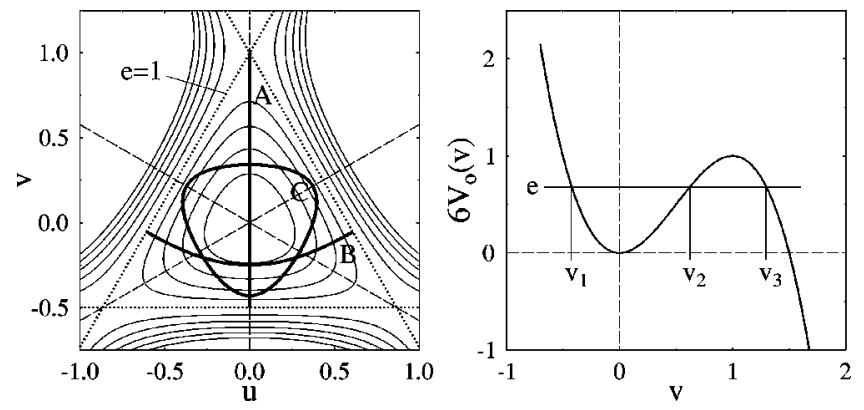

FIG. 6. The Hénon-Heiles potential. Left: equipotential contour lines in scaled energy units $e$ in the plane of scaled variables $u, v$. The dashed lines are the symmetry axes. The three shortest periodic orbits $A, B$, and $C$ (evaluated at the energy $e=1$ ) are shown by the heavy solid lines. Right: cut of the scaled potential along $u=0$.

Using these numerical results we now evaluate the uniform approximation (11) for the joint contribution of the orbits $A, R$, and $L$. The $B$ orbits are included in the standard Gutzwiller approximation, since they stay isolated at all energies and do not interfere with the other orbits. In Fig. 5 the result is shown together with the result of an exact quantummechanical diagonalization done for $\lambda=0.0008$. One can recognize that the uniform approximation tremendously improves over the diverging standard Gutzwiller approximation (dotted line), leading to an excellent agreement with quantum mechanics up to the saddle at $e=1$. Here, as well as in all following comparisons with quantum mechanics, we have coarse grained the density of states by convolution with a Gaussian over an energy interval $\gamma$. In the semiclassical trace formulas this leads [30] to the inclusion of an exponential factor $\exp \left\{-\left(\gamma T_{\xi} / 2 \hbar\right)^{2}\right\}$ in the Gutzwiller amplitude $\mathcal{A}_{\xi}$ of each periodic orbit $\xi$, where $T_{\xi}$ is its period, in regions far enough from the bifurcations for the orbits to be isolated. Note that in the regions between the two bifurcations, the Gutzwiller approximation is not valid, so that our codimension-2 uniform approximation is indispensible.

\section{B. Hénon-Heiles system}

The system of Hénon and Heiles is given by the Hamiltonian [28] $(m=\hbar=1)$

$$
H=\frac{1}{2}\left(p_{x}^{2}+p_{y}^{2}\right)+\frac{1}{2}\left(x^{2}+y^{2}\right)+\lambda\left(x^{2} y-\frac{1}{3} y^{3}\right) .
$$

When the dimensionless scaled variables $u=\lambda x$ and $v=\lambda y$ are introduced, the scaled total energy in units of the saddlepoint energy $E^{*}=1 / 6 \lambda^{2}$ becomes

$$
\begin{aligned}
e=E / E^{*} & =6\left[\frac{1}{2}\left(\dot{u}^{2}+\dot{v}^{2}\right)+V(u, v)\right] \\
& =3\left(\dot{u}^{2}+\dot{v}^{2}\right)+3\left(u^{2}+v^{2}\right)+6 v u^{2}-2 v^{3} .
\end{aligned}
$$

In the left part of Fig. 6 we show the equipotential lines of the potential part of Eq. $(28)$ in the $(u, v)$ plane together with the three shortest periodic orbits $A, B$, and $C$, evaluated at the scaled energy $e=1$. Along the tree mirror axes (dashed lines) the potential is a cubic parabola as shown along $u=0$ in the 

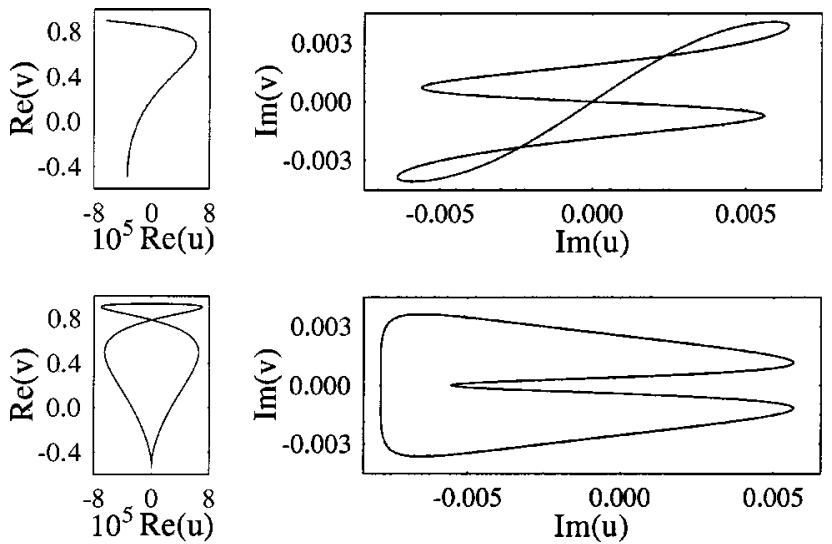

right part of Fig. 6. For an arbitrary energy $e \leqslant 1$, the turning points of the $A$ orbit are determined as the solutions of the equation

$$
e=3 v^{2}+2 v^{3}
$$

and given by

$$
\begin{gathered}
v_{1}=1 / 2-\cos (\pi / 3-\phi / 3), \\
v_{2}=1 / 2-\cos (\pi / 3+\phi / 3), \\
v_{3}=1 / 2-\cos (\phi / 3),
\end{gathered}
$$

with

$$
\cos (\phi)=1-2 e \quad(e \leqslant 1) .
$$

As was shown by Hénon and Heiles, the classical dynamics is quasiregular up to energies of about $e=2 / 3$ and then becomes increasingly chaotic [28]. The $v$ motion of the $A$ orbit with the scaled energy $e_{v}$ is given by [19]

$$
v_{A}^{\left(e_{v}\right)}(t)=v_{1}+\left(v_{2}-v_{1}\right) \operatorname{sn}^{2}(s, q)
$$

in terms of the Jacobi elliptic function [32] $\operatorname{sn}(s, q)$ which depends on the argument $s$ and the modulus $q$, given by

$$
s=t \sqrt{\left(v_{3}-v_{1}\right) / 6} \quad \text { and } \quad q=\sqrt{\frac{v_{2}-v_{1}}{v_{3}-v_{1}}} .
$$

The turning points $v_{i}$ have to be evaluated according to Eqs. (30) with $e=e_{v}$.
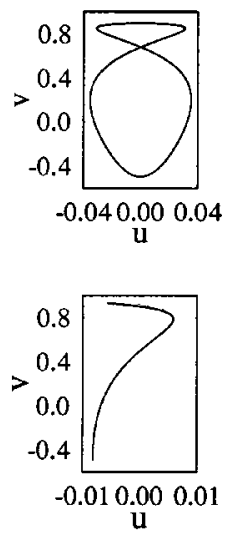

The periodic orbits of the system have been investigated and classified by Churchill et al. [34] as well as Davies et al. [35]. Up to energies of $e \approx 0.97$ there exist only three types of periodic orbits with periods $T$ of the order of $2 \pi$ : the librations $A$ and $B$, and the rotation $C$. Due to the $D_{3}$ symmetry of the potential, orbits $A$ and $B$ occur in three orientations connected by rotations about $2 \pi / 3$ and $4 \pi / 3$ in the $(u$, $v$ ) plane. Orbit $C$ has a degeneracy of 2 because of the time reversal symmetry which corresponds to two different orbits with opposite senses of rotation. The orbit $B$ is unstable for all energies and the orbit $C$ stays stable for energies below $e=0.8922$ where it becomes unstable due to a generic perioddoubling bifurcation. The bifurcation cascades of the $A$ orbit and the orbits generated by them have been studied in detail in Refs. [18,19]; we adapt the names of the orbits given in these references, whereby the subscripts of the orbit names denote their Maslov indices. The $A$ orbit undergoes its first isochronous pitchfork bifurcation at an energy $e_{1}$ $=0.969309$ and the second one at $e_{2}=0.986709$. At the first bifurcation it creates a stable rotational orbit $R_{5}$ which is doubly degenerate due to its two possible senses of rotation. At the second bifurcation, it creates an unstable librational orbit $L_{6}$ which is doubly degenerate due to the reflection symmetry of the potential at the $v$ axis. This scenario repeats itself at higher energies, whereby the pairs of orbits $R_{7}$ and $L_{8}, R_{9}$ and $L_{10}$, etc., are born. The rotational or librational character of these orbits is indicated by the letters $R$ and $L$, respectively. In Fig. 7 the orbits $R_{5}$ and $L_{6}$ are plotted together with their pre-bifurcation complex ghost orbits. All orbits, including $A$, gain one more degeneracy factor 3 due to
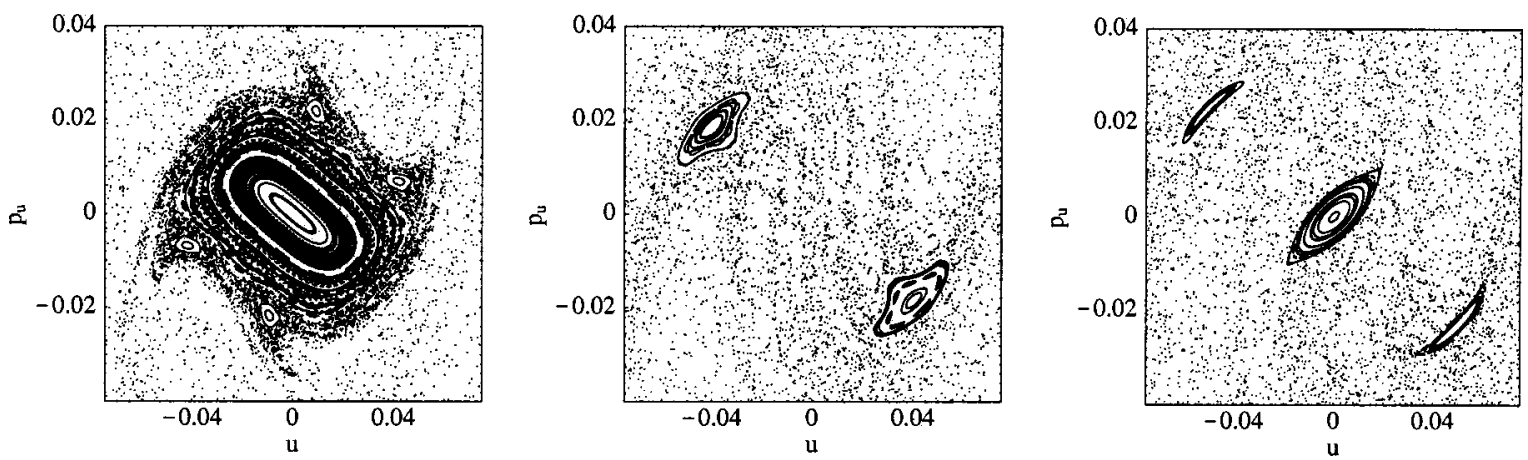

FIG. 8. Poincaré surfaces of section (PSS) of the scaled Hénon-Heiles Hamiltonian (28), taken for $v=0$. Left, $e=0.969$; middle, $e=0.982$; right, $e=0.989$. 

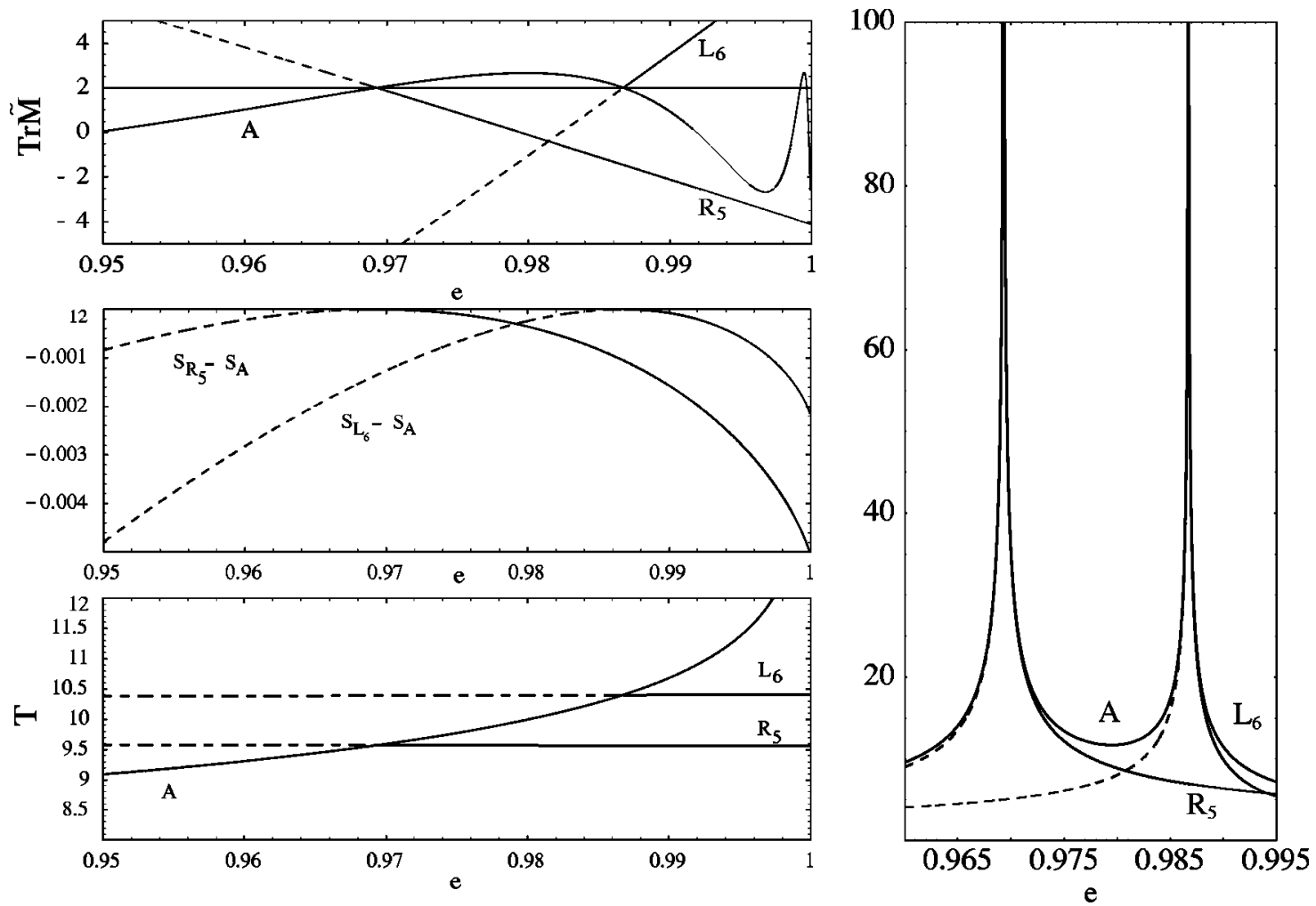

FIG. 9. The same as Fig. 4 for the Hénon-Heiles potential near the first two bifuractions of the $A$ orbit.

the threefold discrete rotational symmetry of the potential, so that the overall degeneracy factors of $A, R$, and $L$ orbits are 3,6 , and 6 , respectively.

In Fig. 8, a part of the PSS for $v=0$ is plotted for energies before the first pitchfork bifurcation (left), between the first and second bifurcation (middle), as well as after the second bifurcation (right). The topology in the vicinity of the bifurcation sequence is correctly described by the normal form (8), as can be seen by a comparison with Fig. 1.

In evaluating the uniform approximation, one can exploit the fact that the actions and the periods of the orbit $A$ can be calculated analytically. The action is given by

$$
\begin{aligned}
S_{A}(E) & =2 \int_{v_{1}}^{v_{2}} \sqrt{e-3 v^{2}+2 v^{3}} d v \\
& =\frac{2}{5 \lambda^{2}} \sqrt{6\left(v_{3}-v_{1}\right)}[E(q)+c K(q)],
\end{aligned}
$$

where the modulus $q$ of the complete elliptic integrals is given in Eq. (33). The constant $c$ is given by

$$
c=-\frac{2}{9}\left(v_{3}-v_{2}\right)\left(2 v_{3}-v_{2}-v_{1}\right)
$$

in terms of the turning points $v_{i}(i=1,2,3)$ given in Eq. (30). The dimensionless period is obtained as

$$
T_{A}(E)=\frac{\partial S_{A}(E)}{\partial E}=2 \sqrt{3} \int_{v_{1}}^{v_{2}} \frac{d v}{\sqrt{e-3 v^{2}+2 v^{3}}}=\frac{2 \sqrt{6}}{\sqrt{v_{3}-v_{1}}} K(q) .
$$

In Fig. 9 the quantities needed to evaluate the uniform approximation (11) of the density of states are shown as a function of the scaled energy $e$. One can see that the stability trace $\operatorname{Tr} \tilde{M}_{A}$ of the $A$ orbit takes on the values +2 at the bifurcation energies. The stability traces of the orbits $R_{5}$ and $L_{6}$ are also plotted; they stay real even for energies $e<e_{1}$ and $e<e_{2}$, respectively, where the two satellites are complex ghost orbits (with their properties shown by dashed lines in Fig. 9).

In Ref. [36], it was shown that the coarse-grained quantum-mechanical density of states of the Hénon-Heiles potential (obtained with a Gaussian smoothing width $\gamma$ $=0.25$ ) can be rather accurately approximated semiclassically, using just the isolated orbits $A, B$, and $C$ and their second repetitions, for energies far enough from the harmonic-oscillator limit $e=0$. In Ref. [12], a uniform approximation for the symmetry breaking at $e=0$ was developed which continuously interpolates from the harmonicoscillator limit, given in Eq. (52) below, to the region where the Gutzwiller trace formula for the isolated orbits is valid. However, the bifurcations of the $A$ orbit have not been treated uniformly in Refs. $[12,36]$, so that the accuracy of the results decreased near the saddle at $e=1$. In Ref. [18] the classical bifurcation cascade in the Hénon-Heiles potential was discussed, in which the sequence of two successive pitchfork bifurcations repeats itself infinitely often.

Presently we test our uniform approximation (11) to the density of states against the quantum-mechanical result obtained for $\lambda=0.03$. The quantum spectrum was, as in Refs. $[12,36]$, obtained by diagonalization of (27) in a finite harmonic-oscillator basis-thus neglecting the effects of 

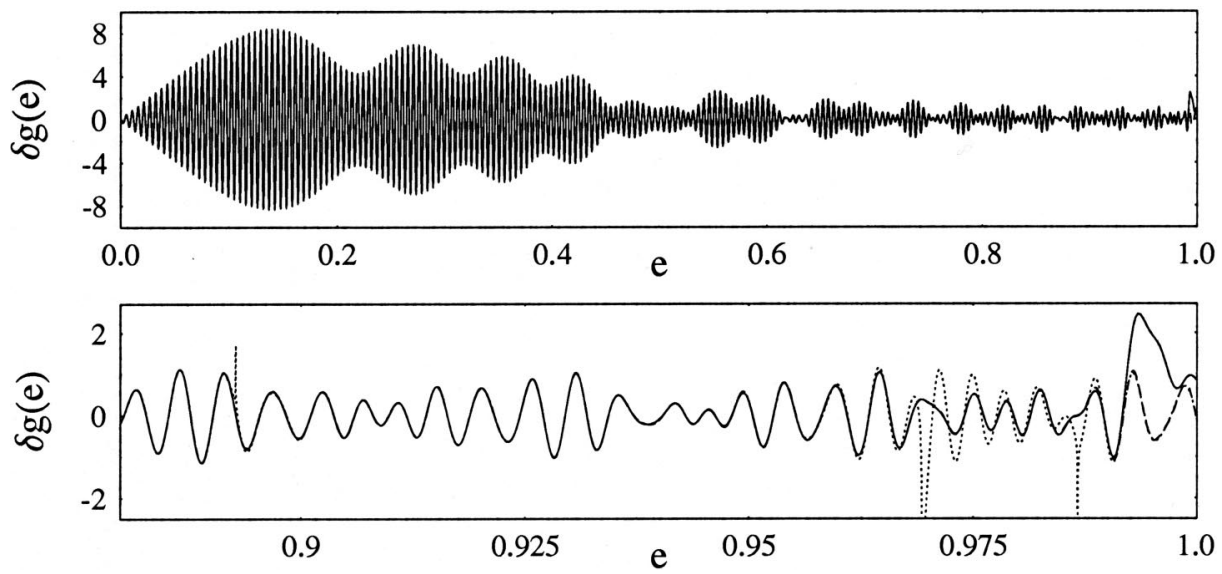

FIG. 10. Oscillating part of density of states in the HénonHeiles potential. Solid lines: quantum-mechanical results obtained for $\lambda=0.03$. Dotted lines: sum of Gutzwiller contributions (6) of all isolated orbits. Dashed lines: codimension-two uniform approximation (11) for the orbits $A, R_{5}$, and $L_{6}$, including orbits $C$ and $D$ in the codimension-1 uniform approximation of Ref. [14] and the isolated $B$ orbit. Coarse graining with Gaussian width $\gamma$ $=0.4$. quantum tunnelling through the barrier. Both quantum and semiclassical results were coarse grained with a Gaussian width of $\gamma=0.4$; for this resolution the inclusion of the second repetitions of all periodic orbits in Eq. (11) was necessary (cf. Ref. [12]). For the pitchfork bifurcation of the second repetition of the orbit $C$ at $e=0.892$, where a doubleloop orbit $D$ is created [36], we used the codimension-1 uniform approximation of Ref. [14]. The upper part of Fig. 10 shows the entire energy region $0 \leqslant e \leqslant 1$, whereas the lower part shows the zoomed region $0.88 \leqslant e \leqslant 1$. The solid lines give the quantum-mechanical result, and the dashed lines the results obtained with our uniform approximation (11) for the first two pitchfork bifurcations of the $A$ orbit. In the region $e \leqslant 0.5$, we have included the uniform approximation for the symmetry breaking, developed in Ref. [12], in order to obtain the correct harmonic oscillator limit for $e$ $\rightarrow 0$. The dotted line in the lower part of the figure corresponds to the sum of the isolated periodic orbits according to the standard Gutzwiller trace formula [1]. Here the divergences due to the lowest bifurcations of the $A$ and $C$ orbits are clearly visible. The uniform result (11), however, exhibits no divergences and its agreement with the quantum result is very satisfactory. The discrepancy arising at $e \gtrsim 0.992$ can be attributed to the influence of the continuum that starts at $e$ $=1$ which was not taken properly into account in our quantum result. In fact, the rather excessive maximum appearing in the latter around $e \sim 0.994$ makes us believe that the latter is erroneous, rather than our semiclassical result. Note that the uniform approximation properly yields the asymptotic Gutzwiller result on either side of the double-pitchfork bifurcation.

In the energy region $e>1$ above the barrier, where the spectrum of the Hénon-Heiles Hamiltonian (27) is continuous, the oscillating part of the density of states is determined by the resonances in the continuum. In order to test the semiclassical periodic orbit theory in this domain, it becomes necessary to calculate both the positions and widths of the resonances. It will then be an interesting question to study which periodic orbits are important in the continuum region. Work along these lines is in progress [37]. Although the continuum region is also classically unbounded, all the $R$ and $L$ orbits bifurcating from the $A$ orbit (which itself ceases to exist above $e=1$ ), as well as the $D$ orbit bifurcating from $C$, continue to exist and are bounded at all energies $e>1$
$[18,35]$. In addition, three new orbits librating across the saddles exist in this region [18,34]; since they have the shortest periods they are expected to play a leading role in the coarse-grained density of states.

\section{Separable Hénon-Heiles system}

The Hénon-Heiles system permits chaotic motion because of the nonseparable term $x^{2} y$ in Eq. (27). Omitting this term one obtains a system which is separable in $x$ and $y$ and hence integrable:

$$
H=\frac{1}{2}\left(p_{x}^{2}+p_{y}^{2}\right)+\frac{1}{2}\left(x^{2}+y^{2}\right)-\frac{\lambda}{3} y^{3} .
$$

Again using dimensionless scaled variables $u=\lambda x$ and $v$ $=\lambda y$ the scaled energy $e$ in units of the saddle point energy $E^{*}$ reads

$$
\begin{aligned}
e & =E / E^{*}=6\left[\frac{1}{2}\left(\dot{u}^{2}+\dot{v}^{2}\right)+V(u, v)\right] \\
& =3\left(\dot{u}^{2}+\dot{v}^{2}\right)+3\left(u^{2}+v^{2}\right)-2 v^{3} .
\end{aligned}
$$

Figure 11 shows a contour plot of the potential part of Eq. (38) in the $(u, v)$ plane together with the two shortest periodic orbits $A$ and $B$ calculated at an energy $e=1$. The two orbits are librations along the $u$ and $v$ axes. The potential along the $v$ axis is the same as that in the right part of Fig. 6,

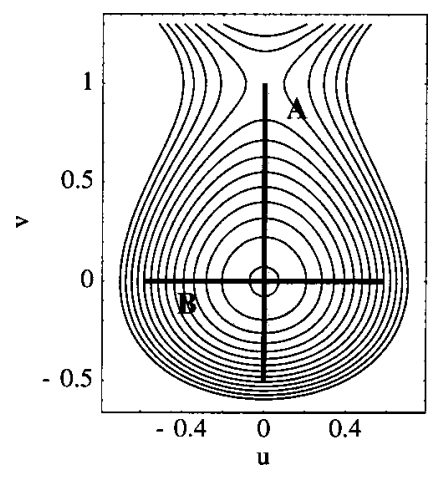

FIG. 11. Equipotential lines in the $(u, v)$ plane for the separable version of the Hénon-Heiles potential. The heavy solid lines show the two shortest periodic orbits $A$ and $B$ evaluated at $e=1$. 

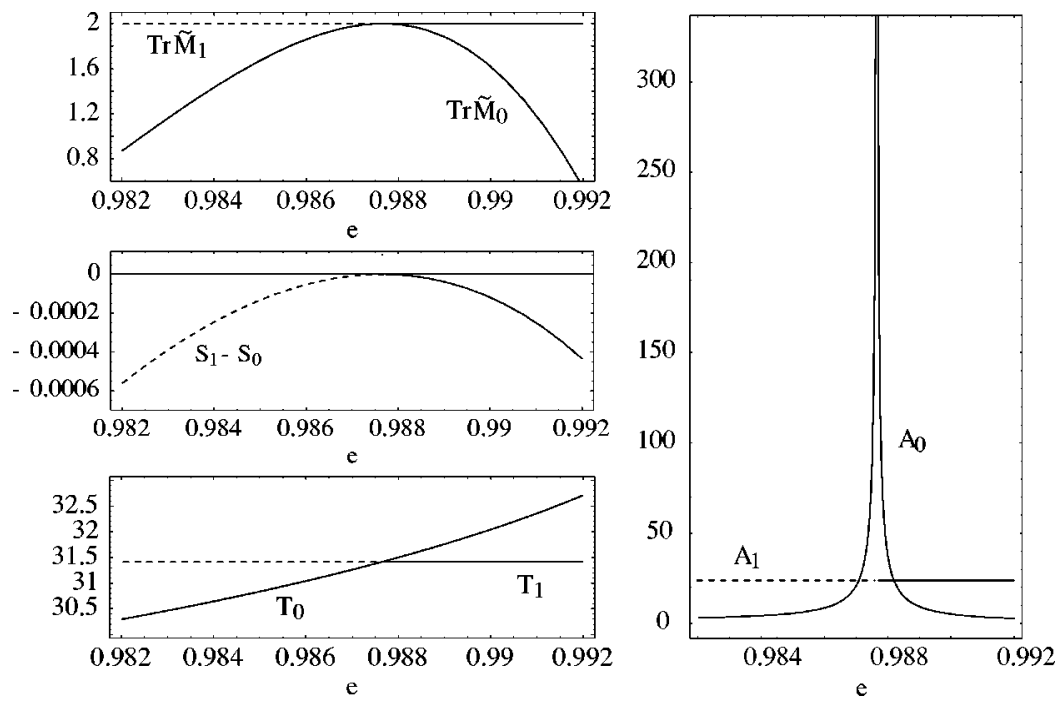

FIG. 12. The same as Fig. 4 for the separable Hénon-Heiles system (37) for the bifurcation of the $k_{u}: k_{v}=5: 3$ resonance at energy $e=0.987655$. The central $A$ orbit is labeled by " 0 ," the bifurcated $5: 3$ torus by " 1. " while the potential along the $u$ axis is harmonic.

Th actions and periods of the $A$ orbit are given by Eqs. (34) and (36), respectively. The trace of its stability matrix is given analytically by [18]

$$
\operatorname{Tr} \tilde{M}_{A}(E)=2 \cos \left[T_{A}(E)\right] .
$$

The $u$ motion of the $B$ orbit is harmonic,

$$
u_{B}(t)=\sqrt{\frac{e_{u}}{3}} \sin (t+\phi),
$$

where $e_{u}$ is the conserved scaled energy in the $u$ direction and the phase $\phi$ is arbitrary. The action and period of the primitive $B$ orbit are those of a harmonic oscillator with frequency $\omega=1$ :

$$
S_{B}(E)=2 \pi E, \quad T_{B}(E)=2 \pi .
$$

The trace of the stability matrix of the $B$ orbit has the constant value $\operatorname{Tr} \tilde{M}_{B}=+2$, which is consistent with its appearing as a torus in the asymptotic analysis given in Appendix C.

The $k_{v}$ th repetition of orbit $A$ bifurcates whenever the condition

$$
\operatorname{Tr} \tilde{M}_{A}^{k_{v}}=2 \cos \left(k_{v} T_{A}\right)=+2
$$

is obeyed, which is equivalent to the resonance condition (cf. Appendix C 2) at the bifurcation energies $E_{b i f}$

$$
k_{v} T_{A}\left(E_{b i f}\right)=2 \pi k_{u}=k_{u} T_{B} .
$$

Thus the bifurcations of the $A$ orbit create the rational tori corresponding to the $k_{v}: k_{u}$ resonances. The new tori form families of degenerate periodic orbits that are related by the $\mathrm{U}(1)$ symmetry due to the freedom in choosing the phase $\phi \in[0,2 \pi)$ in their $u$ motion,

$$
u_{T}(E)=\sqrt{\frac{e-e_{b i f}}{3}} \sin (t+\phi),
$$

where $e_{b i f}$ are the scaled bifurcation energies, while their $v$ motion is "frozen" and identical to that of the $A$ orbit given in Eq. (32) at the corresponding bifurcation energy:

$$
v_{T}(t)=v_{A}^{\left(e_{b i f}\right)}(t) .
$$

The actions of the tori become

$$
S_{T}(E)=k_{v} S_{A}\left(E_{b i f}\right)+k_{u} 2 \pi\left(E-E_{b i f}\right),
$$

so that their periods stay constant at

$$
T_{T}=k_{u} 2 \pi=k_{u} T_{B}
$$

Like for all degenerate orbit families, their stability trace is constant:

$$
\operatorname{Tr} \tilde{M}_{T}=+2 .
$$

We first apply our uniform approximation to the single isolated bifurcation with $k_{u}: k_{v}=5: 3$ which happens at $e$ $=0.987$ 655. In Fig. 12 we show the action difference $S_{1}$ $-S_{0}=S_{T}(E)-S_{A}(E)$, the periods $T_{0}=3 T_{A}(E)$ and $T_{1}=T_{T}$ $=10 \pi$, the traces of the stability matrix, as well as the Gutzwiller and Berry-Tabor amplitudes of the isolated $A$ orbit and the 5:3 torus, respectively. This figure should be compared with Fig. 9 in which the corresponding quantities are shown for the nonintegrable Hénon-Heiles potential. Here the two bifurcations coincide, and instead of the two isolated orbits $R_{5}$ and $L_{6}$ created at the two bifurcations there, we have here only one torus whose stability trace has the constant value +2 .

These quantities are now used to evaluate the uniform approximation for the integrable case, given in Eq. (18). The result is shown in Fig. 13 by the dashed line. It is compared to the exact quantum-mechanical curve (solid line) obtained for $\lambda=0.04$, as well as to the result of including independently the Berry-Tabor contribution of the torus and the Gutzwiller contribution of the isolated $A$ orbit which diverges at the bifurcation (dotted line). All results have been coarse grained by convolution with a Gaussian with smoothing parameter $\gamma=0.1$. We see that the uniform approximation reproduces the quantum result very accurately.

So far, we have discussed and tested our uniform approximations for a double-pitchfork sequence, based on the normal form (8), and its separable limit. In Appendix C, we give an alternative derivation of the uniform approximation for 


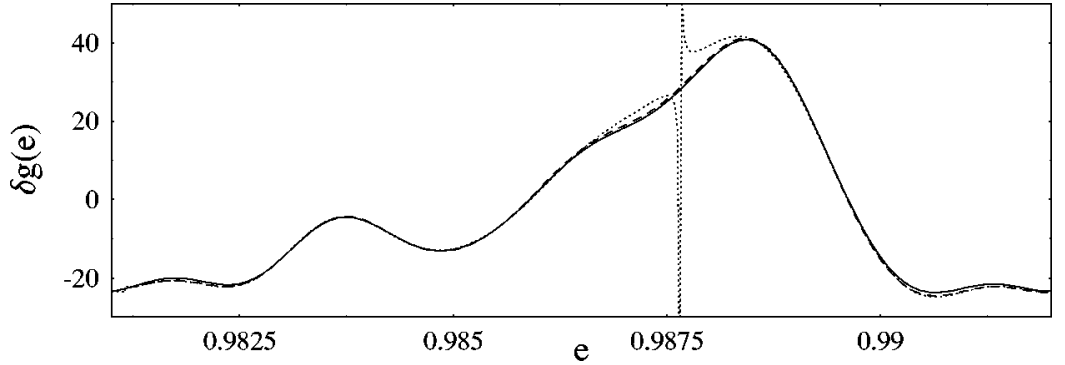

FIG. 13. Oscillating part of level density for the separable Hénon-Heiles system (37) near the 5:3 resonance, coarse-grained with a Gaussian width $\gamma=0.1$. Solid line, quantum-mechanical result obtained with $\lambda=0.04$; dotted line, sum of Berry-Tabor contribution of $5: 3$ torus and Gutzwiller contribution of isolated $A$ orbit; dashed line, uniform approximation (18). the separable limit, starting from the EBK quantization and exploiting the convolution property of the density of states for separable systems. There we do not require any normal form, but we start from a one-dimensional integral (C15) for the density of states which by construction is uniform in the sense that it does not diverge at any energy. By expanding the amplitude and phase functions of the integrand around the bifurcation energies $E_{b i f}$ up to first and second order, respectively, we arrive at approximate integrals which precisely correspond to those obtained from the normal form (8), and which can be reexpressed in terms of the Gutzwiller amplitude of the isolated A orbit and the Berry-Tabor amplitudes of the rational tori. Furthermore, the starting point (C15) allows us also to include the limit $e \rightarrow 0$, in which the amplitude of the isolated $A$ orbit also diverges, in a uniform way.

Since all amplitudes, actions and periods of the isolated $A$ orbit and the tori bifurcating from it can be given analytically for the integrable Hénon-Heites (IHH) potential, it poses no problem to sum over the repetitions of the $A$ orbit and all the tori bifurcating from them. As shown in detail in Appendix $\mathrm{C}$, this leads to the following "grand" uniform approximation which is valid and finite also in the harmonic-oscillator limit $e \rightarrow 0$ :

$$
\begin{aligned}
\delta g_{u n i}(E)= & \sum_{k_{v}=1}^{\infty} \sum_{k_{u}=k_{v}}^{\infty}(-1)^{k_{u}+k_{v}}\left\{\left(A_{A_{k_{u} k_{v}}}(E)\right.\right. \\
& \left.-\frac{1}{2} \sigma_{k_{u} k_{v}} \sqrt{\frac{\hbar}{\pi \Delta S_{k_{u} k_{v}}}} A_{T_{k_{u} k_{v}}}\right) \cos \left[\frac{k_{v}}{\hbar} S_{A}(E)-\frac{\pi}{2}\right] \\
& \left.+\frac{A_{T_{k_{u} k_{v}}} \operatorname{Re}\left[\left(e^{i \pi / 4}\left[1-\delta_{k_{u} k_{v}}\right]+\sqrt{2}\left[C\left(\xi_{k_{u} k_{v}}\right)\right.\right.\right.}{2}\right] \\
& \left.\left.\left.+i S\left(\xi_{k_{u} k_{v}}\right)\right]\right) e^{\left.(i / \hbar) S_{T_{k_{u} k_{v}}}(E)\right]}\right\}+\delta g_{a s}^{(A 0)}(E)+\delta g_{a s}^{(B 0)}(E) .
\end{aligned}
$$

Here we have defined

$$
\begin{gathered}
\mathcal{A}_{A_{k_{u} k_{v}}}(E)=\frac{1}{\pi \hbar} \frac{2 k_{v}\left[T_{A}(E)\right]^{2}}{\left\{\left[k_{v} T_{A}(E)\right]^{2}-\left(2 \pi k_{u}\right)^{2}\right\}}, \\
\sigma_{k_{u} k_{v}}=\operatorname{sgn}\left(E-E_{k_{u} k_{v}}^{*}\right),
\end{gathered}
$$

and

$$
\begin{gathered}
\xi_{k_{u} k_{v}}=\sigma_{k_{u} k_{v}} \sqrt{\frac{2 \Delta S_{k_{u} k_{v}}}{\pi \hbar},} \\
\Delta S_{k_{u} k_{v}}(E)=k_{v} S_{A}(E)-S_{T_{k_{u} k_{v}}}(E) \geqslant 0,
\end{gathered}
$$

and the amplitudes $\mathcal{A}_{T_{k_{u} k_{v}}}$ and actions $S_{T_{k_{u} k_{v}}}$ of the tori are given in Eqs. (C19) and (C20), respectively, of Appendix C.

The first term in Eq. (49) yields, upon summation over all $k_{u}$ and $k_{v}$ and adding the term $\delta g_{a s}^{(A 0)}(E)$ in the last line, precisely the Gutzwiller trace formula (C23) of the isolated $A$ orbit which diverges at the bifurcations and at $E=0$. The second term in the first line is a counter term from the tori that cancels all divergences of the Gutzwiller amplitudes. The second line of Eq. (49) yields the Berry-Tabor trace formula (C18) far away from the bifurcations; near the bifurcations it contains the Stokes factor that interpolates between the Berry-Tabor amplitudes above and zero below the bifurcations, yielding exactly half the Berry-Tabor amplitudes at the bifurcations. The two contributions in the last line of Eq. (49) are small boundary terms, given in Eqs. (C21) and (C24) of Appendix C, which are numerically insignificant but have been included in order to be consistent up to order $\hbar^{-1}$ in the amplitudes.

In the limit $e \rightarrow 0$, where we can neglect all bifurcations, only the diagonal terms with $k_{u}=k_{v}=k$ contribute. The trace formula (49) then leads uniformly to the correct SU(2) harmonic oscillator limit whose trace formula is given in Eq. (C13) of Appendix C (for $\omega=1)$ :

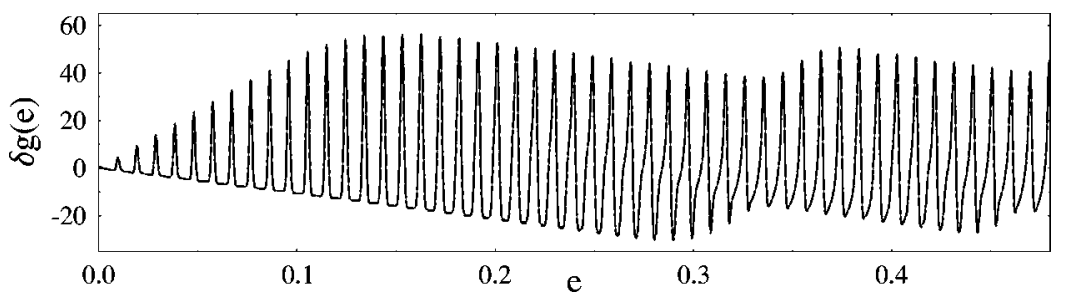

016206-11
FIG. 14. Oscillating part of level density of the separable Hénon-Heiles system (37), coarse grained with $\gamma=0.1$. Solid lines: quantummechanical result for $\lambda=0.04$. Dashed lines: semiclassical results with $k_{u}, k_{v} \leqslant 8$. 


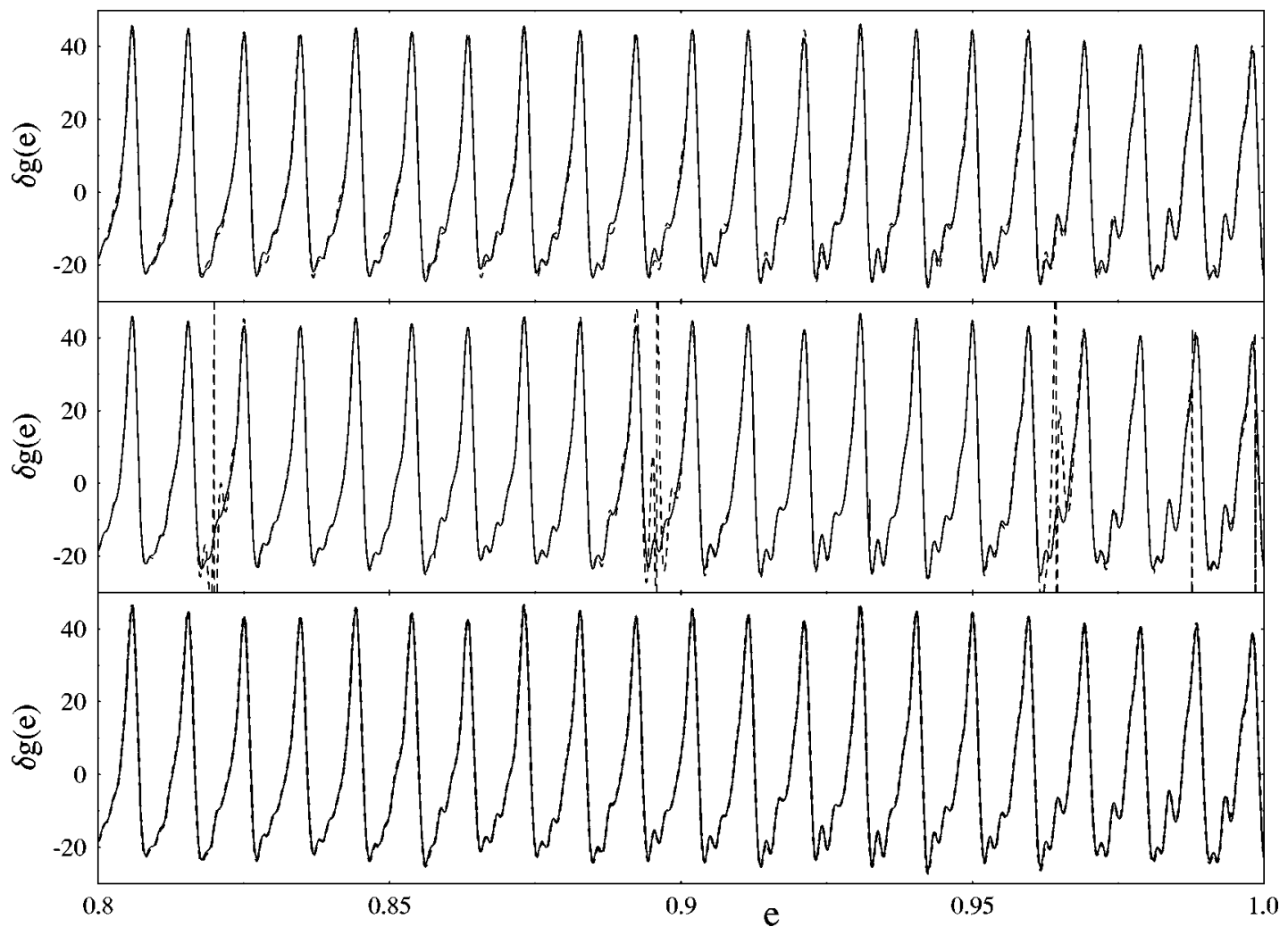

FIG. 15. Oscillating part of level density of the separable Hénon-Heiles system (37), coarse grained with $\gamma=0.1$. Solid lines: quantummechanical result. Dashed lines: semiclassical results with $k_{u}, k_{v} \leqslant 8$. Top: Berry-Tabor result for the tori. Center: sum of Berry-Tabor result for the tori plus Gutzwiller result for the isolated $A$ orbit. Bottom: uniform approximation (49).

$$
\delta g_{\text {uni }}(E) \rightarrow \delta g_{h o}^{i s o}(E)=\frac{2 E}{\hbar^{2}} \sum_{k=1}^{\infty} \cos \left(\frac{k}{\hbar} 2 \pi E\right) \quad(\text { for } e \rightarrow 0) .
$$

(The same limit was obtained in a uniform approximation for the full nonintegrable Hénon-Heiles potential in Ref. [12] neglecting, however, the bifurcations.)

In Figs. 14 and 15, we compare the results obtained from the grand uniform approximation (49) with those of quantum-mechanical calculations for system (38) with $\lambda$ $=0.04$ (with saddle energy $E^{*}=104.666$ corresponding to $e$ $=1$ ), both coarse grained by a Gaussian convolution with an energy range $\gamma=0.1$, including repetition numbers up to $\left|k_{u}\right|,\left|k_{v}\right| \leqslant 8$ into the semiclassical trace formula (49). Figure 14 shows the lowest energy range which exhibits for $e$ $\leq 0.1$ the harmonic-oscillator limit (52) where the amplitude of $\delta g(e)$ is linear in $e$.

In the top panel of Fig. 15 we compare the quantum result to the standard Berry-Tabor trace formula, given in Eq. (C18) of Appendix C, which takes into account only the tori with semiclassical amplitudes proportional to $\hbar^{-3 / 2}$. In the center panel, we have added to them the $A$ orbit contribution described by the Gutzwiller trace formula, given in Eq. (C23) of Appendix C, with amplitudes proportional to $\hbar^{-1}$. The latter is seen to diverge at all bifurcations corresponding to resonances with $k_{u}: k_{v} \geqslant 5: 4$. Between the bifurcations, the result is clearly improved by adding the $A$ orbit contribution and comes very close to the quantum result. In the bottom panel, finally, we show the grand uniform approximation (49) which reproduces the quantum result very well throughout the whole energy region. The bifurcation corresponding to the resonances with $k_{u}: k_{v}=2: 1$ happens at the scaled energy $e=0.998491$; all bifurcations with $k_{u}: k_{v}>2: 1$ happen thus in the top 0.15 percent of the energy scale very near the barrier. In this region, the bifurcations are lying so densely that their independent summation in Eq. (49) is strictly not justified. However, at the present resolution of the spectral density this does not appear to affect our numerical result. On the other hand, the good agreement which we find in Fig. 15 at all lower energies demonstrates that our grand uniform approximation (49) successfully sums all partial bifurcation cascades of the $A$ orbit limited by the repetition numbers 2 $\leqslant k_{v}, k_{u} \leqslant 8$.

We should stress that, like for the nonintegrable HénonHeiles potential, the quantum spectrum was obtained here by diagonalisation in a finite harmonic-oscillator basis. The persistence of our good agreement up to $e \simeq 1$ therefore suggests that the barrier tunneling effects are negligible-at least within the resolution given here by the coarse graining width $\gamma=0.1$.

\section{SUMMARY, CONCLUSIONS, AND OUTLOOK}

We have derived a codimension-2 uniform approximation for the joint contribution of the periodic orbits involved in a 
double pitchfork bifurcation sequence by constructing a suitable normal form. This bifurcation scenario only occurs in systems with discrete symmetries and cannot be treated by the codimension-1 uniform approximations developed in Ref. [14] due to the vicinity of the two pitchfork bifurcations. Furthermore, it does not belong to the unfoldings classified in Ref. [25], so that a different approach became inevitable. We have also studied the limit where both pitchfork bifurcations coincide, resulting in the bifurcation of a torus from an isolated orbit such as it happens in integrable systems. For separable potentials, the same uniform approximation could be rederived from an EBK trace formula that accounts both for an isolated orbit and for the tori bifurcating from it.

Our uniform approximation was tested numerically for two well-known systems with mixed classical dynamics: a double-well potential and the familiar Hénon-Heiles system. In both cases the uniform approximation was shown to reach the asymptotic Gutzwiller approximation on either side of the double-pitchfork bifurcation, while yielding finite amplitudes throughout the whole energy region. The agreement of the semiclassical and quantum-mechanical coarse-grained level densities was found to be excellent.

Our uniform approximation is only valid as long as the considered pair of pitchfork bifurcations is isolated from other bifurcations. In the examples studied here, this is the case for the lowest pair of bifurcations of the isolated $A$ orbit. Since this orbit undergoes an infinite bifurcation cascade cumulating at the barrier energy $e=1$, our approximation will eventually fail for higher double-pitchfork bifurcations; the precise energy where this happens depends on the value of the nonlinearity parameter $\lambda$. However, for the coarsegrained shell structure obtained with a limited resolution (given by a sufficiently large Gaussian width $\gamma$ ), the higher bifurcations become less important and the corresponding divergences in the level density cannot be resolved.

For the separable limit of the Hénon-Heiles system we have obtained analytical expressions for the uniform approximation. This allowed us to sum over a large part of the bifurcation cascade corresponding to rational $k_{u}: k_{v}$ tori with $k_{u}, k_{v} \leqslant 8$. The resulting grand uniform approximation (49) for the semiclassical density of states, which also correctly describes the $\mathrm{SU}(2)$ symmetry-restoring limit for $e \rightarrow 0$, leads to an excellent agreement with the slightly coarse-grained quantum-mechanical density of states even up to the barrier energy $e=1$.

An extension of the semiclassical analysis of the density of states to the energy region above the barrier $(e>1)$, including a rigorous quantum-mechanical determination of the widths and energy shifts due to barrier tunneling in the quasibound region of the spectrum and of the resonances in the continuum region, is in progress [37].

\section{ACKNOWLEDGMENTS}

The authors want to thank P. Schlagheck, H. Schomerus, and T. Bartsch for very helpful discussions and S. Fedotkin and A. Magner for stimulating comments. We thank A. Jung for assistance with the numerical diagonalizations. J.K. ac- knowledges financial support from the Deutsche Forschungsgemeinschaft through the Graduiertenkolleg GRK 638 "Nonlinearity and Nonequilibrium in Condensed Matter."

\section{APPENDIX A: DERIVATION OF THE UNIFORM APPROXIMATION}

In this section we describe the procedure leading to the uniform approximation (11) following the ideas outlined in Ref. [25]. The semiclassical approximation of the density of states is given by

$$
\delta g(E) \approx \frac{1}{2 \pi^{2} \hbar^{2}} \operatorname{Re} \int_{\Omega} d q^{\prime} d p \Psi\left(q^{\prime}, p\right) \exp \left[\frac{i}{\hbar} \Phi\left(q^{\prime}, p\right)-i \frac{\pi}{2} \nu\right]
$$

with a phase function

$$
\Phi\left(q^{\prime}, p\right)=\hat{S}\left(q^{\prime}, p\right)-q^{\prime} p
$$

and an amplitude function

$$
\Psi\left(q^{\prime}, p\right)=\frac{1}{n} \frac{\partial \hat{S}}{\partial E}\left|\frac{\partial^{2} \hat{S}}{\partial q^{\prime} \partial p}\right|^{1 / 2} .
$$

The integration is done over any region $\Omega$ of the PSS and $\nu$ corresponds to the Morse Index. We can set $n=2$ in Eq. (A3) because the nongeneric pitchfork bifurcations are equivalent to generic period-doubling bifurcations [23]. Using the normal form (8) of the generating function the phase function $\Phi$ can be expressed in canonical polar coordinates $I$ and $\phi$ as

$$
\Phi\left(q^{\prime}(\phi, I), p(\phi, I)\right)=S_{0}-\left(\epsilon_{1} \cos ^{2} \phi+\epsilon_{2} \sin ^{2} \phi\right) I-a I^{2}
$$

with

$$
p=\sqrt{2 I} \cos \phi, \quad q^{\prime}=\sqrt{2 I} \sin \phi .
$$

The periodic solutions in Eq. (5) correspond to the stationary points of $\Phi$ at

$$
\frac{\partial \Phi}{\partial \phi}=0, \quad \frac{\partial \Phi}{\partial I}=0,
$$

yielding

$$
\sin (2 \phi)=0, \quad\left(\epsilon_{1} \cos ^{2} \phi+\epsilon_{2} \sin ^{2} \phi\right)+2 a I=0 .
$$

There are four solutions of Eq. (A7): two with $\cos (2 \phi)=1$, corresponding to a satellite orbit which is labeled by 1 in the following, and two with $\cos (2 \phi)=-1$ corresponding to a satellite orbit labeled by 2 . At the stationary points the values of $I$ are

$$
I_{i}=-\frac{\epsilon_{i}}{2 a},
$$

where $i=1,2$. With $\sigma_{i} \equiv \operatorname{sgn}\left(\mathrm{I}_{\mathrm{i}}\right)$ the satellite orbit $i$ is real if $\sigma_{i}=+1$ and represents a ghost solution with complex coordinates $q$ and $p$ if $\sigma_{i}=-1$. The phase function (A4) evaluated at the stationary points (A8) corresponds to the actions of the two satellite orbits, 


$$
S_{i}=S_{0}+\frac{\epsilon_{i}^{2}}{4 a}
$$

The periods are $T_{0}=\partial S_{0} / \partial E$ and

$$
T_{i}=T_{0}+\frac{\epsilon_{i}}{2 a} \frac{\partial \epsilon_{i}}{\partial E}
$$

The traces of the stability matrix can be calculated from

$$
\operatorname{Tr} \tilde{M}=\left(\frac{\partial^{2} \hat{S}}{\partial p \partial q^{\prime}}\right)^{-1}\left[1+\left(\frac{\partial^{2} \hat{S}}{\partial p \partial q^{\prime}}\right)^{2}-\frac{\partial^{2} \hat{S}}{\partial p^{2}} \frac{\partial^{2} \hat{S}}{\partial q^{\prime 2}}\right],
$$

evaluated at the stationary points [13-15]. One obtains

$$
\begin{gathered}
\operatorname{Tr} \tilde{M}_{0}=2-\epsilon_{1} \epsilon_{2}, \quad \operatorname{Tr} \tilde{M}_{1}=2+2 \epsilon_{1} \epsilon_{2}-2 \epsilon_{1}^{2}, \\
\operatorname{Tr} \tilde{M}_{2}=2+2 \epsilon_{1} \epsilon_{2}-2 \epsilon_{2}^{2} .
\end{gathered}
$$

For all orbits the actions (A9), periods (A10), and stabilities (A12) are real quantities even though the orbits themselves can be complex. This characteristic of period-doubling bifurcations which is due to a Stokes transition was already mentioned in Ref. [14].

The Maslov indices $\mu_{i}$ of the periodic orbits are related to the Morse index $\nu$ appearing in Eq. (A1) by

$$
\mu_{i}=\nu+\frac{1}{2}\left(n_{n}-n_{p}\right)
$$

where $n_{n}$ and $n_{p}$ are the number of negative and positive eigenvalues of the matrix

$$
\Phi^{\prime \prime}=\left(\begin{array}{cc}
\frac{\partial^{2} \Phi}{\partial q^{\prime 2}} & \frac{\partial^{2} \Phi}{\partial q^{\prime} \partial p} \\
\frac{\partial^{2} \Phi}{\partial q^{\prime} \partial p} & \frac{\partial^{2} \Phi}{\partial p^{2}}
\end{array}\right)
$$

evaluated at the stationary points. They follow as

$$
\begin{gathered}
\mu_{0}=\nu+\left[\operatorname{sgn}\left(\epsilon_{1}\right)+\operatorname{sgn}\left(\epsilon_{2}\right)\right] / 2, \\
\mu_{1}=\nu+\left[\operatorname{sgn}\left(\epsilon_{2}-\epsilon_{1}\right)-\operatorname{sgn}\left(\epsilon_{1}\right)\right] / 2, \\
\mu_{2}=\nu+\left[\operatorname{sgn}\left(\epsilon_{1}-\epsilon_{2}\right)-\operatorname{sgn}\left(\epsilon_{2}\right)\right] / 2 .
\end{gathered}
$$

For the amplitude function the following ansatz was found to be sufficient:

$$
\Psi(\phi, I)=\alpha_{0}+\alpha_{1} I+\alpha_{2} I^{2}
$$

Equation (A1), expressed in canonical coordinates, now takes on the following form:

$$
\begin{aligned}
\delta g(E) \approx & \frac{1}{4 \pi^{2} \hbar^{2}} \operatorname{Re} \exp \left[\frac{i}{\hbar} S_{0}-i \frac{\pi}{2} \nu\right] \\
& \times \int_{0}^{2 \pi} d \phi \int_{0}^{\infty} d I\left(\alpha_{0}+\alpha_{1} I+\alpha_{2} I^{2}\right) \\
& \times \exp \left\{-\frac{i}{\hbar}\left[\left(\epsilon_{1} \cos ^{2} \phi+\epsilon_{2} \sin ^{2} \phi\right) I+a I^{2}\right]\right\} .
\end{aligned}
$$

The parameters $\epsilon_{i}$ measure the distance to the bifurcation $i$. They are given by the actions (A9) of the new born orbits as

$$
\epsilon_{i}=-2 \tilde{\sigma}_{i} \sigma_{i} \sqrt{\left|\Delta S_{i}\right|},
$$

where we have set

$$
a=\widetilde{\sigma}_{i} \equiv \operatorname{sgn}\left(\Delta S_{i}\right) .
$$

In order to achieve a uniform approximation one evaluates Eq. (A19) in stationary-phase approximation at the stationary points which yields

$$
\begin{aligned}
\delta g^{(S P)}(E)= & \frac{1}{\pi \hbar} \frac{\left(\alpha_{0}+\alpha_{1} I_{i}+\alpha_{2} I_{i}^{2}\right)}{\sqrt{\left|\operatorname{det} \Phi^{\prime \prime}\left(I_{i}\right)\right|}} \\
& \times \cos \left\{\frac{1}{\hbar}\left[S_{0}-\left(\epsilon_{1} \cos ^{2} \phi_{i}+\epsilon_{2} \sin ^{2} \phi_{i}\right) I_{i}-a I_{i}^{2}\right]\right\} .
\end{aligned}
$$

One can now determine the coefficients $\alpha_{0}, \alpha_{1}$ and $\alpha_{2}$ by identifying the Gutzwiller amplitudes $\mathcal{A}_{i}$ with

$$
\mathcal{A}_{i}=\frac{1}{\pi \hbar} \frac{\left(\alpha_{0}+\alpha_{1} I_{i}+\alpha_{2} I_{i}^{2}\right)}{\sqrt{\left|\operatorname{det} \Phi^{\prime \prime}\left(I_{i}\right)\right|}}
$$

where $i=1,2,3$. Defining

$$
\widetilde{\epsilon}(\phi) \equiv \epsilon_{1} \cos ^{2} \phi+\epsilon_{2} \sin ^{2} \phi,
$$

the integrals with respect to $I$ in Eq. (A19) can be calculated analytically using

$$
\begin{aligned}
F_{n} & \equiv \int_{0}^{\infty} d I I^{n} e^{-(i / \hbar)\left[\widetilde{\epsilon}(\phi) I+a I^{2}\right]} \\
& =\left(i \hbar \frac{\partial}{\partial \widetilde{\epsilon}(\phi)}\right)^{n} \int_{0}^{\infty} d I e^{-(i / \hbar)\left[\widetilde{\epsilon}(\phi) I+a I^{2}\right]} .
\end{aligned}
$$

They yield for $n=0,1$, and 2 the results given in Eqs. (12) and (13). The remaining $\phi$ integral over the interval $[0,2 \pi]$ can easily be calculated numerically.

\section{Appendix B: Derivation of the uniform approximation for the separable limit}

In the case $\epsilon_{1}=\epsilon_{2} \equiv \epsilon$ the phase function (A4) simplifies to

$$
\Phi\left(q^{\prime}(\phi, I), p(\phi, I)\right)=S_{0}-\epsilon I-a I^{2}
$$

and becomes independent of $\phi$, corresponding to an integrable system. The stationary point of $\Phi$ corresponds to a torus with the radial coordinate 


$$
I_{T}=-\frac{\epsilon}{2 a}
$$

With the definition

$$
\sigma \equiv \operatorname{sgn}\left(I_{T}\right),
$$

the torus is real if $\sigma=+1$, while it is imaginary if $\sigma=-1$ which can be understood from

$$
I=\frac{p^{2}+q^{\prime 2}}{2} .
$$

The action of the torus becomes

$$
S_{T}=S_{0}+\frac{\epsilon^{2}}{4 a},
$$

and for the period one obtains

$$
T_{T}=T_{0}+\frac{\epsilon}{2 a} \frac{\partial \epsilon}{\partial E} .
$$

Using Eq. (A11) one finds that $\operatorname{Tr} \tilde{M}=+2$ which is characteristic of an orbit family. The amplitude function can be derived from Eq. (A18), resulting in

$$
\Psi(I)=\alpha_{0}+\beta I,
$$

with

$$
\beta=\alpha_{1}-\frac{\epsilon}{2 a} \alpha_{2}
$$

using $\alpha_{0}, \alpha_{1}$, and $\alpha_{2}$ from Eq. (A18). This can be seen by the following integration by parts,

$$
\begin{aligned}
& -\frac{\epsilon}{2 a} \int_{0}^{\infty} d I I \exp \left(\frac{i}{\hbar} \Phi\right) \\
& =\frac{1}{2 a} \int_{0}^{\infty} d I I \exp \left(\frac{i}{\hbar} \Phi\right)(-\epsilon-2 a I+2 a I) \\
& =\frac{1}{2 a} \int_{0}^{\infty} d I I \exp \left(\frac{i}{\hbar} \Phi\right)\left(\frac{\partial \Phi}{\partial I}+2 a I\right) \\
& =\int_{0}^{\infty} d I I^{2} \exp \left(\frac{i}{\hbar} \Phi\right)+\mathcal{O}(\hbar),
\end{aligned}
$$

where the integral that was neglected in the last step is of relative order $\hbar$. Thus we obtain, to leading order in $\hbar$, the result (B7).

The functions in Eq. (A24) no longer depend on $\phi$ so that the integration over $\phi$ can be performed giving a factor of $2 \pi$. The remaining expression for $\delta g(E)$ then has the form

$$
\begin{aligned}
\delta g(E)= & \frac{1}{2 \pi \hbar^{2}} \operatorname{Re} \exp \left[\frac{i}{\hbar} S_{0}-i \frac{\pi}{2} \nu\right] \int_{0}^{\infty} d I\left(\alpha_{0}+\beta I\right) \\
& \times \exp \left[-\frac{i}{\hbar}\left(\epsilon I+a I^{2}\right)\right] .
\end{aligned}
$$

Exactly the same formula can be found in Ref. [15] in relation with a special case of a generic period-quadrupling bi- furcations. Using the integrals (12) and (13), one arrives at

$$
\begin{aligned}
\delta g(E)= & \frac{1}{\pi \hbar} \frac{\beta}{2 a} \cos \left(\frac{S_{0}}{\hbar}-\frac{\pi}{2}(\nu+1)\right) \\
& +\frac{1}{\pi \hbar^{3 / 2}} \sqrt{\frac{\pi}{2|a|}\left(\alpha_{0}-\frac{\beta \epsilon}{2 a}\right)} \\
& \times \operatorname{Re}\left(e ^ { ( i / \hbar ) ( S _ { 0 } + \epsilon ^ { 2 } / 4 a ) - i ( \pi / 2 ) \nu } \left\{\frac{e^{-i(\pi / 4) \tilde{\sigma}}}{\sqrt{2}}\right.\right. \\
& \left.\left.+\sigma\left[C\left(\sqrt{\frac{\epsilon^{2}}{2 \pi \hbar|a|}}\right)-i \tilde{\sigma} S\left(\sqrt{\frac{\epsilon^{2}}{2 \pi \hbar|a|}}\right)\right]\right\}\right) .
\end{aligned}
$$

It remains to express all parameters by the quantities that enter into the asymptotic contributions of the torus and the central orbit. A stationary phase approximation of Eq. (B11) would deliver the contribution of the stationary point corresponding to the torus only. In order to obtain the contribution of the central periodic orbit at $I=0$ one has to include also the end-point corrections to the stationary phase approximation (cf. Appendix C 2). This amounts to an asymptotic expansion of the Fresnel functions for large arguments $x \gg 1$ (cf. Ref. [32]). Keeping their two leading terms,

$$
\begin{aligned}
& C(x) \sim \frac{1}{2}+\sin \left(\pi x^{2} / 2\right), \\
& S(x) \sim \frac{1}{2}-\cos \left(\pi x^{2} / 2\right),
\end{aligned}
$$

leads to

$$
\begin{aligned}
\delta g(E)= & \frac{1}{\pi \hbar} \frac{\alpha_{0}}{|\epsilon|} \cos \left(\frac{S_{0}}{\hbar}-\frac{\pi}{2}[\nu+\operatorname{sgn}(\epsilon)]\right) \\
& +\frac{1}{\pi \hbar^{3 / 2}} \frac{1+\sigma}{2} \sqrt{\frac{\pi}{|a|}}\left(\alpha_{0}-\frac{\beta \epsilon}{2 a}\right) \\
& \times \cos \left[\frac{S_{0}+\frac{\epsilon^{2}}{4 a}}{\hbar}-\frac{\pi}{2}\left(\nu+\frac{\tilde{\sigma}}{2}\right)\right] .
\end{aligned}
$$

Two asymptotic contributions can be recognized. One is of the order $\hbar^{-1}$, corresponding to the central periodic orbit, and one is of the order $\hbar^{-3 / 2}$ which is the torus contribution. Asymptotically one obtains a torus contribution only on the real side of the bifurcation $\sigma=+1$ whereas on the complex side $\sigma=-1$ the torus contribution asymptotically vanishes even though the torus amplitude itself must not necessarily go to zero. The fact that it still gives no contribution asymptotically is due to a Stokes transition of the torus.

Expressing now Eq. (B11) with the Gutzwiller amplitude $A_{A}$ of the central orbit,

$$
\mathcal{A}_{0}=\mathcal{A}_{A}=\frac{1}{\pi \hbar} \frac{\alpha_{0}}{|\epsilon|},
$$

and the Berry-Tabor amplitude of the torus, 


$$
\mathcal{A}_{T}=\frac{1}{\pi \hbar^{3 / 2}} \sqrt{\frac{\pi}{|a|}}\left(\alpha_{0}-\frac{\beta \epsilon}{2 a}\right),
$$

and setting $\Delta S \equiv S_{T}-S_{0}$ as well as $\nu=\mu_{0}-\operatorname{sgn}(\epsilon)$ finally yields the uniform approximation (18).

\section{APPENDIX C: ALTERNATIVE DERIVATION OF THE UNIFORM APPROXIMATION FOR THE SEPARABLE LIMIT FROM EBK QUANTIZATION}

In this appendix we give an alternative derivation of the uniform approximation for the separable limit, starting from EBK (or WKB) quantization in one dimension and using the fact that the two-dimensional density of states can be obtained by a convolution of the two one-dimensional densities of state. We present the general formula in the first subsection and derive from it the known exact trace formula for harmonic oscillators. In the second subsection we specialize to the integrable Hénon-Heiles system and present a uniform trace formula which sums over all bifurcations and leads to the correct harmonic-oscillator limit for $e \rightarrow 0$.

\section{Semiclassical trace formula for separable Hamiltonians}

For a separable Hamiltonian in two dimensions $(u, v)$,

$$
H=H_{u}\left(u, p_{u}\right)+H_{v}\left(v, p_{v}\right)=E_{u}+E_{v},
$$

the Schrödinger equation separates

$$
\begin{gathered}
\hat{H} \Phi_{n m}(u, v)=E_{n m} \Phi_{n m}(u, v), \\
\Phi_{n m}(u, v)=\phi_{n}(u) \psi_{m}(v), \quad E_{n m}=\epsilon_{n}+\varepsilon_{m},
\end{gathered}
$$

and the exact quantum density of states can be written as a convolution integral over the two level densities of the onedimensional systems:

$$
g(E)=\int_{0}^{E} g_{u}\left(E-E^{\prime}\right) g_{v}\left(E^{\prime}\right) d E^{\prime},
$$

where

$$
g_{u}(E)=\sum_{n} \delta\left(E-\epsilon_{n}\right), \quad g_{v}(E)=\sum_{m} \delta\left(E-\varepsilon_{m}\right),
$$

and we have assumed $\varepsilon_{m}, \epsilon_{n}>0$. We now use EBK quantization $\left(i=u, v ; n_{u}=n, n_{v}=m\right)$,

$$
H_{i}=H\left(I_{i}\right), \quad I_{i}=\frac{1}{2 \pi} S_{i}=\frac{1}{2 \pi} \oint_{p_{i}} d q_{i}=\hbar\left(n_{i}+1 / 2\right),
$$

and Poisson summation (cf. Refs. [4,30]) to obtain the following semiclassical trace formula for each of the onedimensional level densities:

$$
g_{i}(E)=\frac{T_{i}(E)}{2 \pi \hbar} \sum_{k_{i}=-\infty}^{\infty}(-1)^{k_{i}} \cos \left[\frac{k_{i}}{\hbar} S_{i}(E)\right],
$$

which is identical to the Gutzwiller trace formula [1] for a one-dimensional system and yields the corresponding EBK
(WKB) spectrum. Using Eq. (C3), we thus get the twodimensional trace formula for the separable Hamiltonian (C1),

$$
\begin{aligned}
g(E)= & \frac{1}{(2 \pi \hbar)^{2}} \sum_{k_{u}, k_{v}=-\infty}^{\infty}(-1)^{k_{u}+k_{v}} \\
& \times \int_{0}^{E} T_{u}\left(E-E^{\prime}\right) T_{v}\left(E^{\prime}\right) \cos \left[\frac{k_{u}}{\hbar} S_{u}\left(E-E^{\prime}\right)\right] \\
& \times \cos \left[\frac{k_{v}}{\hbar} S_{v}\left(E^{\prime}\right)\right] d E^{\prime} .
\end{aligned}
$$

If the convolution integral is done exactly, this trace formula yields the spectrum $E_{n m}$ in Eq. (C2) in the EBK approximation. The contribution from $k_{u}=k_{v}=0$ yields the average Thomas-Fermi (TF) level density which becomes a simple convolution integral over the primitive periods of the two one-dimensional motions:

$$
\widetilde{g}(E)=g_{T F}(E)=\frac{1}{(2 \pi \hbar)^{2}} \int_{0}^{E} T_{u}\left(E-E^{\prime}\right) T_{v}\left(E^{\prime}\right) d E^{\prime} .
$$

The semiclassical trace formula (C7), which contains the smooth part (C8), requires only the classical periods $T_{i}(E)$ and actions $S_{i}(E)$ of the one-dimensional systems as an input. Nevertheless, it contains all information about the periodic orbits of the two-dimensional system-not only the degenerate families forming two-dimensional rational tori, but also the existing isolated orbits as will be shown explicitly in the following. It also handles all possible bifurcations uniformly. The formula (C7) therefore goes far beyond the standard trace formulas $[4,7]$ for integrable systems which only take the leading rational tori into account and cannot account for bifurcations.

The integral in Eq. (C7) can, in general, not be done analytically. For a harmonic oscillator,

$$
H=\frac{1}{2}\left(p_{u}^{2}+p_{v}^{2}\right)+\frac{1}{2}\left(\omega_{u}^{2} u^{2}+\omega_{v}^{2} v^{2}\right),
$$

we have $S_{i}(E)=2 \pi E / \omega_{i}$ and $T_{i}(E)=2 \pi / \omega_{i}(i=u, v)$. The integral then is elementary and yields

$$
\begin{aligned}
g_{h o}(E)= & \frac{1}{2 \pi \hbar \omega_{u} \omega_{v}} \sum_{k_{u}, k_{v}=-\infty}^{\infty}(-1)^{k_{u}+k_{v}} e^{-i \pi / 2} \\
& \times\left\{\frac{1}{\left(k_{u} / \omega_{u}-k_{v} / \omega_{v}\right)} e^{i k_{u} 2 \pi E / \hbar \omega_{u}}\right. \\
& \left.+\frac{1}{\left(k_{v} / \omega_{v}-k_{u} / \omega_{u}\right)} e^{-i k_{v} 2 \pi E / \hbar \omega_{v}}\right\} .
\end{aligned}
$$

For irrational frequency ratios $\omega_{u}: \omega_{v}$, no singularities arise and using the identity [32] 


$$
\frac{1}{\sin (z)}=\sum_{k=-\infty}^{\infty} \frac{(-1)^{k}}{(z-k \pi)} \quad(z \neq n \pi)
$$

we can sum the first term in Eq. (C10) over all $k_{v}$ and the second term over all $k_{u}$. The result is the exact Gutzwiller trace formula for the irrational harmonic oscillator $[30,40]$ which yields its correct quantum-mechanical spectrum,

$$
\begin{aligned}
g_{h o}(E)= & \frac{E}{\hbar \omega_{u} \hbar \omega_{v}}+\frac{1}{\hbar \omega_{u}} \sum_{k_{u}=1}^{\infty} \frac{(-1)^{k_{u}}}{\sin \left(k_{u} \pi \frac{\omega_{v}}{\omega_{u}}\right)} \sin \left(k_{u} \frac{2 \pi E}{\hbar \omega_{u}}\right) \\
& +\frac{1}{\hbar \omega_{v}} \sum_{k_{v}=1}^{\infty} \frac{(-1)^{k_{v}}}{\sin \left(k_{v} \pi \frac{\omega_{u}}{\omega_{v}}\right)} \sin \left(k_{v} \frac{2 \pi E}{\hbar \omega_{v}}\right) .
\end{aligned}
$$

The last two terms contain the sums over the (only) isolated periodic orbits along the $u$ and $v$ axes. Corresponding trace formulas for rational frequency ratios can be obtained from the above by taking suitable limits [40]. E.g., in the isotropic limit $\omega_{u}=\omega_{v}=\omega$ one obtains

$$
g_{h o}^{(i s o)}(E)=\frac{E}{(\hbar \omega)^{2}}\left\{1+2 \sum_{k=1}^{\infty} \cos \left(k \frac{2 \pi E}{\hbar \omega}\right)\right\},
$$

which is again a quantum-mechanically exact trace formula in terms of the twofold degenerate families of periodic orbits with $\mathrm{SU}(2)$ symmetry, having the primitive actions $S(E)$ $=2 \pi E / \omega$. Note that the standard methods to derive the trace formula for integrable systems [4,7] cannot be used for harmonic oscillators, since the ("curvature") tensor of second derivatives of the Hamiltonian (C9) with respect to the torus actions $I_{i}=E_{i} / \omega_{i}$ is identically zero.

For systems in which the actions $S_{i}(E)$ are no simple functions, the integral in Eq. (C7) can in general only be done numerically. This becomes practically impossible if one wants to generate the semiclassical EBK spectrum by summing (C7) over all $k_{u}$ and $k_{v}$. In the example treated in the next subsection, we show how an asymptotic evaluation of the integral can be used to establish the relation to the BerryTabor type trace formula for the tori and the Gutzwiller trace formula for isolated $A$ orbit, and to derive the same uniform approximation for the bifurcations of the isolated $A$ orbit as we have obtained in Appendix B using the normal form theory.

\section{Asymptotic evaluation for the separable Hénon-Heiles system and global uniform approximation}

In the following we specialize to the integrable HénonHeiles (IHH) system (37), expressed in the scaled coordinates $u, v$ as in Eq. (38). Here $T_{u}=T_{B}=2 \pi, S_{u}(E)=S_{B}(E)$ $=2 \pi E$, and the period $T_{A}(E)$ and action $S_{A}(E)$ of the $v$ motion are given in Eqs. (34)-(36). The TF level density is then given by

$$
g_{T F}(E)=\frac{1}{2 \pi \hbar^{2}} S_{A}(E)
$$

and the oscillating part can be written as

$$
\begin{aligned}
\delta g(E)= & \frac{1}{2 \pi \hbar^{2}} \sum_{k_{u}, k_{v}=-\infty}^{\infty}(-1)^{k_{u}+k_{v}} \\
& \times \int_{0}^{E} T_{A}\left(E_{v}\right) e^{i\left[k_{v} S_{A}\left(E_{v}\right)+2 \pi k_{u}\left(E-E_{v}\right)\right] / \hbar} d E_{v},
\end{aligned}
$$

where the prime indicates that the TF contribution from $k_{u}$ $=k_{v}=0$ must be left out. Note that upon independent summations over $k_{u}$ and $k_{v}$ the imaginary parts cancel, consistently with the general real expression (C7).

In order to evaluate the integral in Eq. $(\mathrm{C} 15)$ in the semiclassical limit $\hbar \rightarrow 0$, we use the following general formula [38,39]:

$$
\begin{aligned}
\int_{a}^{b} T(x) e^{i S(x) / \hbar} d x \sim & \sum_{i} T\left(x_{i}\right) \sqrt{\frac{2 \pi \hbar}{\left|S^{\prime \prime}\left(x_{i}\right)\right|}} e^{i S\left(x_{i}\right) / \hbar+i \operatorname{sgn}\left[S^{\prime \prime}\left(x_{i}\right)\right] \pi / 4} \\
& +T(b) \frac{\hbar}{S^{\prime}(b)} e^{i S(b) / \hbar-i \pi / 2} \\
& +T(a) \frac{\hbar}{S^{\prime}(a)} e^{i S(a) / \hbar+i \pi / 2}
\end{aligned}
$$

neglecting corrections of higher order in $\hbar$. Equation (C16) is a generalization of the standard stationary-phase integration, taking into account the end-point corrections whose contributions are of order $\hbar^{1 / 2}$ relative to those from the stationary points. The sum in the first line above is to be taken over all stationary points $x_{i}$ which lie in the integration interval $a$ $\leqslant x_{i} \leqslant b$. If either of the end points $a$ or $b$ is a stationary point, its contribution to the sum has to be divided by two and the corresponding term in the second line above has to be omitted. If there is no stationary point at all in the interval $[a, b]$, there is no contribution to the first line and the leading terms of the integral $I$ are of order $\hbar$ as given by the endpoint contributions in the second line alone.

The stationarity condition for the phase in the integral (C15) leads to the resonance condition for the rational tori,

$$
k_{v} T_{A}\left(E_{k_{u} k_{v}}^{*}\right)=2 \pi k_{u}=k_{u} T_{B}
$$

Note that this condition is independent of the energy $E$. The stationary points $E_{v}=E_{k_{u} k_{v}}^{*}$ are the energies at which the $k_{v}$ th repetition of the $A$ orbit bifurcates, cf. Eqs. (42) and (43) in Sec. IV C. The condition (C17) can only be fulfilled if $k_{u}$ and $k_{v}$ have the same sign and if $\left|k_{u}\right| \geqslant\left|k_{v}\right|$. In most formulas below, we take $k_{u}$ and $k_{v}$ to be positive (or zero for one of them) and account for the two signs by an extra factor of 2 in the summations, taking real parts where necessary. For $k_{u}$ $=k_{v}=k$, the stationary point is at $E_{k k}^{*}=0$, for all other tori the stationary points are at finite energies. This gives, according to the first line in Eq. (C16), the following asymptotic contribution to lowest order in $\hbar$ : 


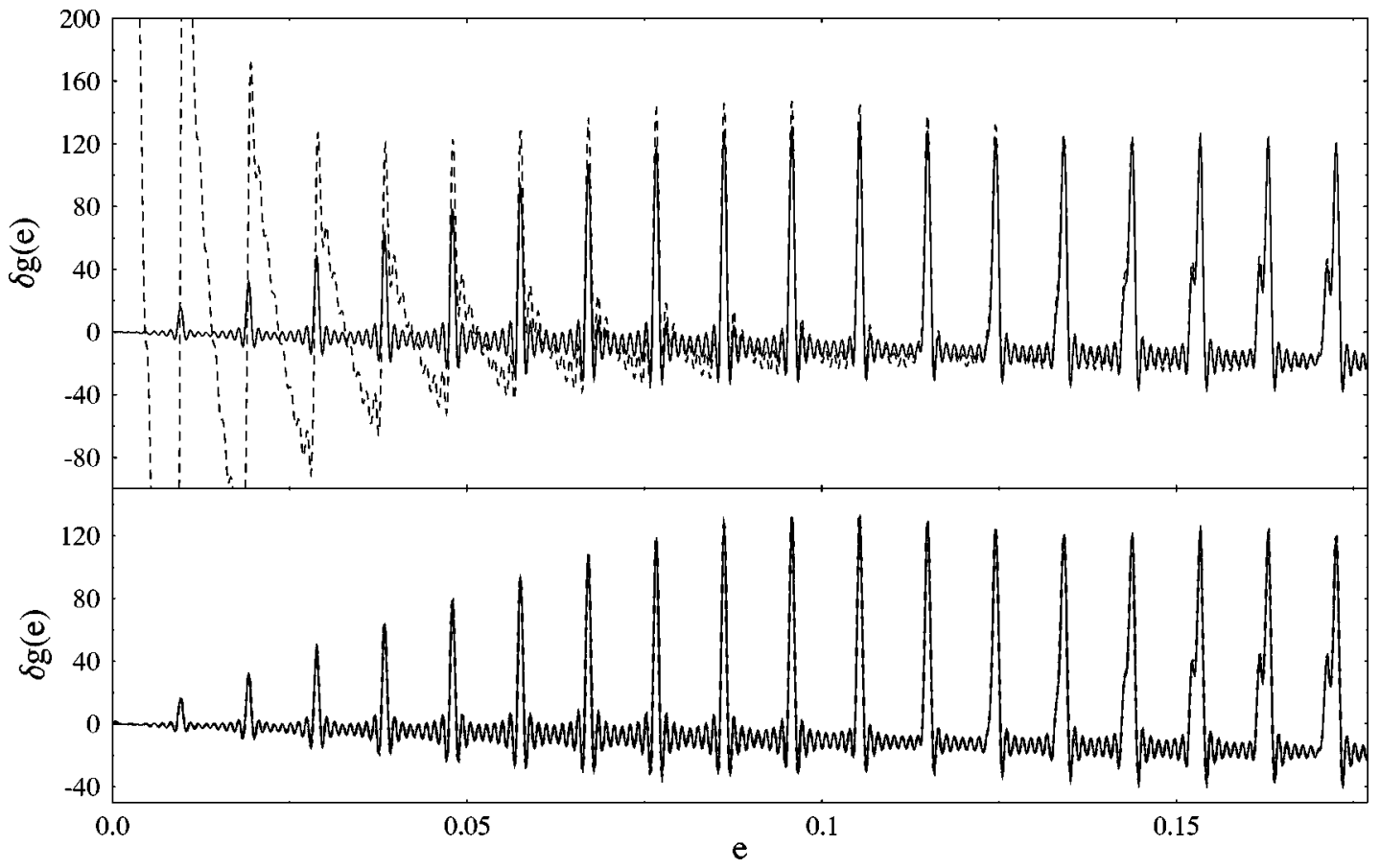

FIG. 16. Shell structure in the level density of the integrable Hénon-Heiles system. Solid lines: exact EBK integral (C15). Dashed lines: asymptotic approximations. Upper panel: Berry-Tabor contributions (C18) of the $T$ tori plus Gutzwiller contribution (C23) of the isolated $A$ orbit. Lower panel: uniform approximation (49). In both cases, repetition numbers $\left|k_{u}, k_{v}\right| \leqslant 8$ are included. Here the lowest energy region is shown where the isolated $A$ orbit contribution diverges in the limit $e \rightarrow 0$.

$$
\delta g_{a s}^{(T)}(E)=\sum_{k_{v}=1}^{\infty} \sum_{k_{u}=k_{v}}^{\infty} A_{T_{k_{u} k_{v}}} \cos \left[\frac{1}{\hbar} S_{T_{k_{u} k_{v}}}(E)+\frac{\pi}{4}\right] \Theta\left(E-E_{k_{u} k_{v}}^{*}\right),
$$

which is exactly the Berry-Tabor trace formula $[4,7]$ with the amplitudes

$$
\begin{gathered}
A_{T_{k_{u} k_{v}}}=f_{k_{u} k_{v}} \frac{(-1)^{k_{u}+k_{v}}}{\hbar^{3 / 2}} \frac{k_{u}}{k_{v}} \sqrt{\frac{2 \pi}{k_{v} T_{A}\left(E_{k_{u} k_{v}}^{*}\right)}}, \\
f_{k_{u} k_{v}}=\left\{\begin{array}{lll}
2 & \text { for } & k_{u} \neq k_{v} \\
1 & & k_{u}=k_{v}
\end{array}\right\} .
\end{gathered}
$$

The actions of the tori are

$$
S_{T_{k_{u} k_{v}}}(E)=k_{v} S_{A}\left(E_{k_{u} k_{v}}^{*}\right)+2 \pi k_{u}\left(E-E_{k_{u} k_{v}}^{*}\right) \quad\left(E \geqslant E_{k_{u} k_{v}}^{*}\right) .
$$

The diagonal torus $T_{k k}$, which comes from the lower end point $E_{v}=0$ of the integral in Eq. (C15), corresponds to the $k$ th repetition of the classical $B$ orbit. This is somewhat puzzling, since classically this orbit appears to be isolated along the $u$ axis, but semiclassically it contributes in the same way as the two-dimensional tori with $k_{u} \neq k_{v}$ with an amplitude proportional to $\hbar^{-3 / 2}$. The reason for this is connected to the fact that the energy $E_{k k}^{*}=0$ at which it bifurcates is simultaneously the limit of the isotropic two-dimensional harmonic oscillator in which all orbits form a two-dimensionally degenerate family with $\mathrm{SU}(2)$ symmetry. The contributions from $k_{v}=0$ and $k_{u} \neq 0$ lead to a small correction,

$$
\delta g_{a s}^{(B 0)}(E)=\frac{1}{\pi \hbar} \sum_{k_{u}=1}^{\infty} \frac{(-1)^{k_{u}}}{k_{u}} \sin \left(\frac{k_{u}}{\hbar} 2 \pi E\right),
$$

which is of order $\sqrt{\hbar}$ with respect to Eq. (C19) and found to be negligible in our numerical calculations.

The upper end point $E_{v}=E$ of the integral in Eq. (C15) corresponds to motion along the $v$ axis which classically gives the isolated $A$ orbit. According to the second line in Eq. (C16), this yields the asymptotic contribution

$$
\delta g_{\text {as }}^{(A)}(E)=\frac{T_{A}(E)}{\pi \hbar} \sum_{k_{v}=1}^{\infty} \sum_{k_{u}=-\infty}^{\infty} \frac{(-1)^{k_{u}+k_{v}}}{\left[k_{v} T_{A}(E)-2 \pi k_{u}\right]} e^{i\left[k_{v} S_{A}(E) / \hbar-\pi / 2\right]} .
$$

Using the identity (C11) we can do the summation over $k_{u}$ analytically and find

$$
\delta g_{a s}^{(A)}(E)=\frac{T_{A}(E)}{2 \pi \hbar} \sum_{k_{v}=1}^{\infty} \frac{(-1)^{k_{v}}}{\sin \left[k_{v} T_{A}(E) / 2\right]} \cos \left[\frac{k_{v}}{\hbar} S_{A}(E)-\frac{\pi}{2}\right],
$$

which is exactly the Gutzwiller trace formula for the isolated $A$ orbit. Of course, this expression cannot be used at the bifurcation energies of the $A$ orbit where, on one hand, Eq. (C11) is not valid and, on the other hand, the upper end-point correction from the integral (C15) should be replaced by one-half of the corresponding torus contribution to Eq. (C18). The contributions from $k_{u}=0$ and $k_{v} \neq 0$ in Eq. (C22) lead to a small correction, 


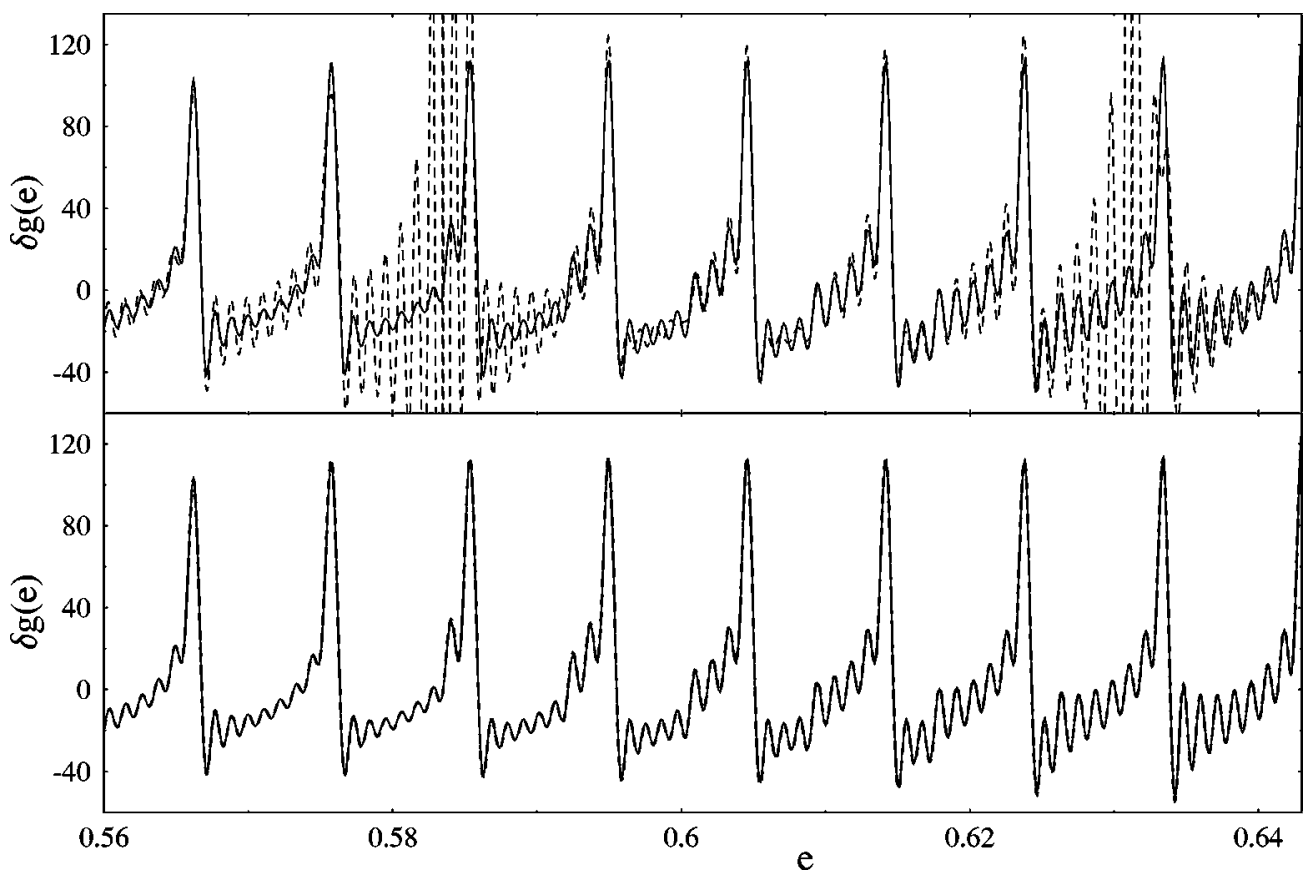

FIG. 17. The same as Fig. 16 in an intermediate energy region. The top panel exhibits the divergence of the Gutzwiller contributions of the $A$ orbit (dashed line) near the $k_{u}: k_{v}=9: 8$ and $8: 7$ resonances.

$$
\delta g_{a s}^{(A 0)}(E)=\frac{1}{\pi \hbar} \sum_{k_{v}=1}^{\infty} \frac{(-1)^{k_{v}}}{k_{v}} \sin \left(\frac{k_{v}}{\hbar} S_{A}(E)\right),
$$

which is included in Eqs. (C22) and (C23) and will be referred to below.

We have thus established that the isolated $A$ orbit emerges asymptotically, with its standard Gutzwiller amplitude [41], from the upper end-point corrections of the EBK trace for- mula (C15), whereas the tori with their standard Berry-Tabor amplitudes come from the stationary points of the phase in the integral (C15).

In order to obtain finite amplitudes at the bifurcations and the symmetry point $e=0$, we have to develop a uniform approximation. This can be done quite easily by expanding the phase and the amplitude of the integrand in Eq. (C15) around the stationary points $E_{k_{u} k_{v}}^{*}$ up to first and second order in $E_{v}-E_{k_{u} k_{v}}^{*}$, respectively. Noting that the torus action $S_{T}(E)$ in

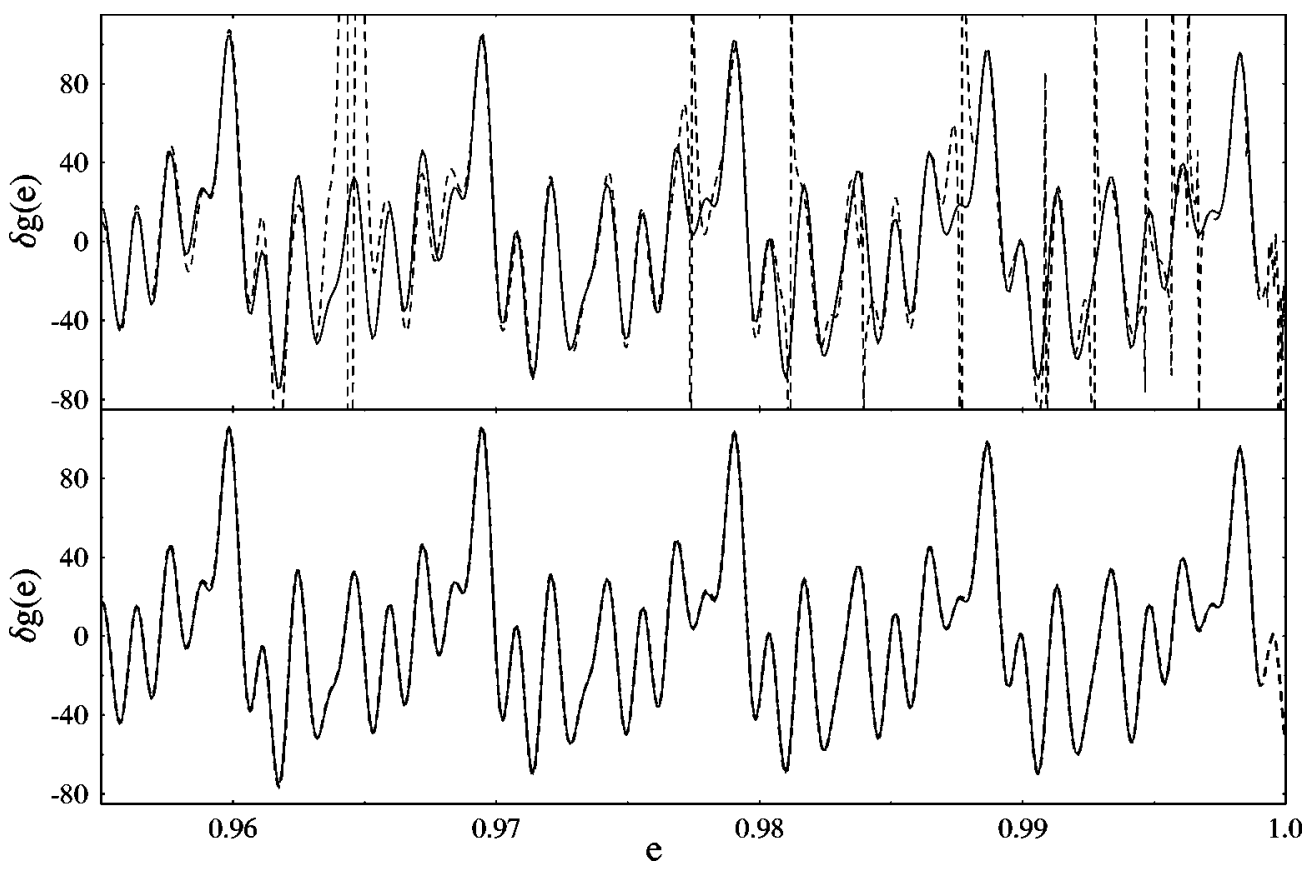

FIG. 18. The same as Fig. 16 in the top energy range, covering the resonances with $k_{u}: k_{v} \geqslant 3: 2$. 
Eq. (C20) represents the first two terms of the same expansion of $S_{A}(E)$ around $E-E_{k_{u} k_{v}}^{*}$, this leads to the approximate contributions

$$
\begin{gathered}
\operatorname{Re}\left\{e^{(i / \hbar) S_{T_{k_{u} k_{v}}}(E)-i \pi\left(k_{u}+k_{v}\right)} \int_{0}^{E}\left(b_{k_{u} k_{v}}+c_{k_{u} k_{v}} E_{v}\right)\right. \\
\left.\times e^{(i / 2 \hbar) a_{k_{u} k_{v}}\left(E_{v}-E_{k_{u} k_{v}}^{*}\right)^{2}} d E_{v}\right\},
\end{gathered}
$$

which are exactly of the same type as those which we have derived from the normal form theory in Appendix B, and which can be integrated analytically using the formulas (12) and (13). The parameters $a_{k_{u} k_{v}}, b_{k_{u} k_{v}}$, and $c_{k_{u} k_{v}}$ must be determined by the requirement that the asymptotic amplitudes and actions of both the $A$ orbit and the $T_{k_{u} k_{v}}$ torus be recovered far away from the bifurcation energy $E_{k_{u} k_{v}}$. This procedure is completely analogous to what has been discussed in the earlier appendixes and need not be repeated here. The final uniform approximation which we obtain after summing over all tori is given in Eq. (49).

The uniform trace formula (49) is discussed and tested versus quantum-mechanical results in Sec. IV C. Here we compare its results with those of a numerical integration of the EBK trace formula (C15). We choose the value $\lambda=0.04$, where the saddle energy is $E=104.666$, and a resolution of the energy spectrum limited by $\left|k_{u}\right|,\left|k_{v}\right| \leqslant 8$. In Figs. 16-18, covering different energy regions, the upper panels show the results of Eq. (C15) by the solid lines and the sum of the asymptotic Berry-Tabor contributions of the tori (C18) plus the Gutzwiller contribution (C23) of the isolated $A$ orbit by the dashed lines. In the lower panel, the same results of Eq. (C15) (solid lines) are compared to those of the uniform approximation (49) (dashed lines). In all cases, the latter proves to be an excellent approximation to the exactly integrated EBK trace formula (C15).

In Fig. 16 the lowest energy region is shown, where the Gutzwiller contributions of the $A$ orbit are seen to diverge in the limit $e \rightarrow 0$. The divergences disappear in the uniform approximation. In Fig. 17, an intermediate energy region is shown which includes the divergences of the isolated $A$ contributions at the bifurcations of the $k_{u}: k_{v}=9: 8$ and 8:7 resonances. Figure 18 shows the top energy region containing all resonances with $k_{u}: k_{v} \geqslant 3: 2$.

These results demonstrate that the uniform approximation (49), which expresses the level density in terms of the BerryTabor and Gutzwiller amplitudes of the periodic orbits, reproduces the numerically integrated EBK trace formula (C15) to a high degree of accuracy.

Finally we stress that our above derivation of the uniform approximation (49) is not limited to the separable HénonHeiles system, but can easily be modified to any separable potential by starting from the general EBK trace formula (C7) rather than from Eq. (C15).
[1] M. C. Gutzwiller, J. Math. Phys. 12, 343 (1971).

[2] M. C. Gutzwiller, Chaos in Classical and Quantum Mechanics (Springer, New York, 1990).

[3] V. M. Strutinsky and A. G. Magner, Elem. Part. \& Nucl. (Atomizdat, Moskva) 7, 356 (1976) [Sov. J. Part. Nucl. 7, 138 (1976)].

[4] M. V. Berry and M. Tabor, Proc. R. Soc. London, Ser. A 349, 101 (1976); J. Phys. A 10, 371 (1977).

[5] R. Balian and C. Bloch, Ann. Phys. (N.Y.) 69, 76 (1972).

[6] V. M. Strutinsky, A. G. Magner, S. R. Ofengenden, and T. Døssing, Z. Phys. A 283, 269 (1977).

[7] S. C. Creagh and R. G. Littlejohn, Phys. Rev. A 44, 836 (1991); J. Phys. A 25, 1643 (1992).

[8] A. M. Ozorio de Almeida and J. H. Hannay, J. Phys. A 20, 5873 (1987).

[9] A. M. Ozorio de Almeida, Hamiltonian Systems: Chaos and Quantization (Cambridge University Press, Cambridge, England, 1988).

[10] S. C. Creagh, Ann. Phys. (N.Y.) 248, 60 (1996).

[11] S. Tomsovic, M. Grinberg, and D. Ullmo, Phys. Rev. Lett. 75, 4346 (1995); D. Ullmo, M. Grinberg, and S. Tomsovic, Phys. Rev. E 54, 135 (1996).

[12] M. Brack, P. Meier, and K. Tanaka, J. Phys. A 32, 331 (1999).

[13] M. Sieber, J. Phys. A 29, 4715 (1996).

[14] H. Schomerus and M. Sieber, J. Phys. A 304537 (1997).

[15] M. Sieber and H. Schomerus, J. Phys. A 31, 165 (1998).

[16] J.-M. Mao and J. B. Delos, Phys. Rev. A 45, 1746 (1992).

[17] J. Main, G. Wiebusch, A. Holle, and K. H. Welge, Phys. Rev.
Lett. 57, 2789 (1996).

[18] M. Brack, Found. Phys. 31, 209 (2001).

[19] M. Brack, M. Mehta, and K. Tanaka, J. Phys. A 34, 8199 (2001).

[20] K. R. Meyer, Trans. Am. Math. Soc. 149, 95 (1970).

[21] A. D. Bruno, Math. USSR. Sb. 12, 271 (1970); (unpublished).

[22] For a recent review, see also P. Leboeuf and A. Mouchet, Ann. Phys. (N.Y.) 275, 54 (1999).

[23] H. Then, diploma thesis, University of Ulm, 1999; H. Then and M. Sieber (unpublished).

[24] H. Schomerus, Europhys. Lett. 38, 423 (1997); H. Schomerus and F. Haake, Phys. Rev. Lett. 79, 1022 (1997).

[25] H. Schomerus, J. Phys. A 31, 4167 (1998).

[26] D. A. Sadovskii, J. A. Shaw, and J. B. Delos, Phys. Rev. Lett. 75, 2120 (1995); D. A. Sadovskii and J. B. Delos, Phys. Rev. E 54, 2033 (1996).

[27] M. J. Feigenbaum, J. Stat. Phys. 19, 25 (1978); Physica D 7, 16 (1983).

[28] M. Hénon and C. Heiles, Astron. J. 69, 73 (1964).

[29] M. V. Berry and K. E. Mount, Rep. Prog. Phys. 35, 315 (1972).

[30] M. Brack and R. K. Bhaduri Semiclassical Physics, Frontiers in Physics No. 96 (Westview Press, Bolder, 2003).

[31] V. M. Strutinsky, Nucl. Phys. A 122, 1 (1968).

[32] M. Abramowitz and I. A. Stegun, Handbook of Mathematical Functions, 9th printing (Dover, New York, 1970).

[33] P. J. Richens, J. Phys. A 15, 2110 (1982).

[34] R. C. Churchill, G. Pecelli, and D. L. Rod, in Stochastic Be- 
havior in Classical and Quantum Hamiltonian Systems, edited by G. Casati and J. Ford (Springer-Verlag, New York, 1979), p. 76.

[35] K. T. R. Davies, T. E. Huston, and M. Baranger, Chaos 2, 215 (1992)

[36] M. Brack, R. K. Bhaduri, J. Law, and M. V. N. Murthy, Phys. Rev. Lett. 70, 568 (1993); M. Brack, R. K. Bhaduri, J. Law, M. V. N. Murthy, and Ch. Maier, Chaos 5, 317 (1995); 5, 707(E) (1995).

[37] J. Kaidel, P. Winkler, and M. Brack (unpublished); J. Kaidel,
Ph.D. thesis, University of Regensburg, 2003, http:// www.opus-bayern.de/uni-regensburg/volltexte/2004/349

[38] A. Erdélyi, Asymptotic Expansions (Dover, New York, 1956).

[39] R. Wong, Asymptotic Approximation of Integrals (Academic Press, San Diego, 1989).

[40] M. Brack and S. R. Jain, Phys. Rev. A 51, 3462 (1995).

[41] The same result was obtained by P. J. Richens, J. Phys. A 15, 2101 (1982), starting from the action-angle variables for a general two-dimensional integrable system containing an isolated orbit. 